Finance and Economics Discussion Series Divisions of Research \& Statistics and Monetary Affairs Federal Reserve Board, Washington, D.C.

\title{
Jump-Diffusion Processes and Affine Term Structure Models: Additional Closed-Form Approximate Solutions, Distributional Assumptions for Jumps, and Parameter Estimates
}

\section{J. Benson Durham}

2005-53

NOTE: Staff working papers in the Finance and Economics Discussion Series (FEDS) are preliminary materials circulated to stimulate discussion and critical comment. The analysis and conclusions set forth are those of the authors and do not indicate concurrence by other members of the research staff or the Board of Governors. References in publications to the Finance and Economics Discussion Series (other than acknowledgement) should be cleared with the author(s) to protect the tentative character of these papers. 
Jump-Diffusion Processes and Affine Term Structure Models:

Additional Closed-Form Approximate Solutions, Distributional Assumptions for Jumps, and Parameter Estimates

\author{
J. Benson Durham* \\ Division of Monetary Affairs \\ Board of Governors of the Federal Reserve System \\ Washington, DC 20551 \\ (202) 452-2896 \\ j.benson.durham@frb.gov
}

\begin{abstract}
Affine term structure models in which the short rate follows a jump-diffusion process are difficult to solve, and the parameters of such models are hard to estimate. Without analytical answers to the partial difference differential equation (PDDE) for bond prices implied by jumpdiffusion processes, one must find a numerical solution to the PDDE or exactly solve an approximate PDDE. Although the literature focuses on a single linearization technique to estimate the PDDE, this paper outlines alternative methods that seem to improve accuracy. Also, closed-form solutions, numerical estimates, and closed-form approximations of the PDDE each ultimately depend on the presumed distribution of jump sizes, and this paper explores a broader set of possible densities that may be more consistent with intuition, including a bi-modal Gaussian mixture. GMM and MLE of one- and two-factor jump-diffusion models produce some evidence for jumps, but sensitivity analyses suggest sizeable confidence intervals around the parameters.
\end{abstract}

\footnotetext{
${ }^{*}$ With no implication whatsoever, the author thanks Sanjiv Das, Henrik Rasmussen, and seminar participants at the Federal Reserve Board for helpful comments and suggestions on this project. The views presented are solely those of the author and do not necessarily represent those of the Federal Reserve Board or its staff.
} 


\section{Introduction}

Shortcomings in the Black-Scholes-Merton model of option prices are well documented. One possible relaxation of the model's restrictions, first proposed by Merton (1976), is to incorporate the possibility that, in addition to diffusion, the underlying asset jumps discontinuously. ${ }^{1}$ Such models that incorporate Brownian increments as well as potential nonlocal instantaneous price changes have been applied in the academic literature (and presumably in practice also) to derivatives written on equities (Merton, 1976; Ball and Torous, 1983) and foreign exchange (Bates, 1996) as well as fixed-income instruments and the underlying yield curve (Ahn and Thompson, 1988; Baz and Das, 1996; Das, 2002; Johannes, 2004; Piazzesi, 2005). However, determining whether a particular asset price movement is a jump or a manifestation of a diffusion process is difficult. Indeed, whether a pure Gaussian or a jumpdiffusion process better captures interest rate movements is an open empirical question. ${ }^{2}$ But this issue aside, this paper focuses on the implications of expanding standard affine term structure models (ATSMs) to include jumps with respect to the derivation of solutions to the bond pricing equation and the estimation of parameters.

Regarding the issue of solutions to ATSMs, jump-diffusion processes for the short rate imply a (second-order parabolic) partial difference differential equation (PDDE) for bond prices that does not necessarily have a closed-form solution. In the absence of an analytical answer, one must find numerical solutions to the PDDE or solve an approximate PDDE exactly. This paper addresses the literature on the second of these approaches (Ahn and Thompson, 1988; and Baz and Das, 1996) in two general respects. First, although existing studies focus on a single linearization technique to estimate the PDDE, the following proposes alternative methods that under reasonable parameterizations seem to improve accuracy. Second, closed-form solutions, numerical estimates, and closed-form approximations of the PDDE each ultimately depend on the key assumption regarding the distributional characteristics of jump sizes, and the current literature on linearization techniques exclusively examines the case in which jumps are normally distributed. This paper explores a broader set of densities, including the exponential/Bernoulli

\footnotetext{
${ }^{1}$ Press (1967) was perhaps the first to explore the hypothesis that (log) stock price changes follow a Poisson mixture of normal distributions.

${ }^{2}$ Das (2002) and Johannes (2004) present empirical cases for interest rate models that incorporate jumps, and more generally, Aït-Sahalia (2004) finds that it is possible to disentangle Brownian noise from jumps.
} 
distribution from Das and Foresi (1996), which permits useful comparisons with an analytical solution, as well as a bi-modal Gaussian mixture developed in this paper. ${ }^{3}$

Regarding parameter estimation, this paper considers a wider variety of distributional assumptions for jump size and employs simple variants of the general method of moments (GMM) and maximum likelihood estimation (MLE). Also, the MLE uses both direct proxies for the short rate, as with GMM, and indirect proxies consisting of observable zero-coupon bond yields. Using the closed-form solutions to approximate PDDEs for bond prices, one can express the latent factor(s) in terms of observable variables(s), broadly consistent with Pearson and Sun (1994). In general, the data do seem to provide some evidence that the short-rate follows a jump-diffusion process, but with respect to application of the broader model to pricing bonds, the Gaussian and jump parameters seem sensitive to estimation technique as well as the proxy used for the short rate (where applicable).

Section 2 reviews the derivation of the PDDE implied by ATSMs that include a jumpdiffusion process for the short rate. Section 3 summarizes the existing literature on current linearization techniques to approximate the PDDE, outlines alternative approximations, and describes simple parameterizations of the model under the common assumption that jump follows a (uni-modal) Gaussian distribution. Section 4 examines alternative assumptions regarding the distribution of jump size and also summarizes relevant (arbitrary) parameterizations. Section 5 describes the parameter estimates following GMM and MLE, and Section 6 outlines the derivation and the parameter estimation of a two-factor jump-diffusion ATSM in which the second state variable is the (time-varying) central tendency of the short rate. Section 7 concludes.

\section{The Bond Pricing Equation under Jump-Diffusion}

Of course, relaxation of the assumption that (underlying) financial assets follow pure diffusion processes considerably complicates the derivation of option and bond price formulae, but one can nonetheless incorporate jumps and obtain tractable approximations for pricing. For example, Merton (1976) derives a semi-closed-form solution for the price of a call option on a

\footnotetext{
${ }^{3}$ The section on GMM estimation also considers the possibility that jumps follow a Bernoulli mixture of uniform distributions.
} 
stock that involves an infinite series of weighted Black-Scholes solutions ${ }^{4}$ in which the weights are effectively the probabilities associated with the realization of the number of jumps drawn from a Poisson distribution. Turning to bonds, this paper focuses on the case of a jumpaugmented Vasicek (1977) model. ${ }^{5}$ To derive the bond-pricing equation, assume that the instantaneous (unobservable) short rate, $r$, follows

$$
d r=a(b-r) d t+\sigma d W+J d \pi(h)
$$

where $a$ is the mean reversion coefficient, $b$ is the "target" interest rate or central tendency of the short rate, $t$ is time, $\sigma$ is the instantaneous volatility coefficient, $d W$ is the Weiner increment, $J$ is the jump size, $d \pi$ is the increment in a Poisson process with intensity rate $h$, and $\tau=T-t$ (time to maturity). Under the assumption of a single factor, bond prices, $P(r, \tau)$, are a function of the short rate and time to maturity, and their dynamics, assuming that all time dependence comes through the state variable, $r$, and using Ito's lemma for jump-diffusion processes, follow

$$
d P=\left(\frac{\partial P}{\partial r} a(b-r)+\frac{1}{2} \sigma^{2} \frac{\partial^{2} P}{\partial r^{2}}\right) d t+\sigma \frac{\partial P}{\partial r} d W+[P(r+J, t)-P(r, t)] d \pi .
$$

Following the standard capital asset pricing relation (Cochrane, 2001; Das and Foresi, 1996), the holding period return of the bond should be equal to the risk-free rate plus a term premium, which with trivial rearranging follows

$$
E_{t}\left[\frac{d P}{P}\right]-\left(\frac{1}{P} \frac{\partial P}{\partial \tau}+r\right) d t=-E_{t}\left[\frac{d \Lambda}{\Lambda} \frac{d P}{P}\right],
$$

and the dynamics of the pricing kernel, $\Lambda$, follow

\footnotetext{
${ }^{4}$ That is, Merton solves the jump-augmented Black-Scholes equation exactly, albeit conditional on the number of jumps. In this formulation, the spot price in the pure Gaussian case, $S_{0}$, corresponds to $S_{0} e^{(\mu-r) \tau} J^{N(\tau)}$, where $\mu$ is the drift of the stock, $J$ is expressed as a multiple of the stock price, and $N$ is the number of jumps until expiry. Central to this derivation is the fact that one can derive a convenient expression for the terminal distribution of $S$ from the jump-augmented process,

$$
\frac{d S}{S}=\mu d t+\sigma d W+(J-1) d \pi(h) .
$$

${ }^{5}$ Ahn and Thompson (1988) examine an alternative case of a jump-augmented Cox, Ingersoll, and Ross (1985) (CIR) model.
} 


$$
\frac{d \Lambda}{\Lambda}=-r d t-\lambda d W{ }^{6}
$$

Note that the market price of interest rate diffusion risk is constant and equal to $\lambda$ and that this expression reflects Merton’s (1976) assumption that the market price of jump risk is unsystematic and thus diversifiable. ${ }^{7}$ Plugging the dynamics of bond prices (2) and the pricing kernel (4) into the equation for holding period return (3), we arrive at the fundamental PDDE for bond prices,

$$
0=[a(b-r)-\lambda \sigma] \frac{\partial P}{\partial r}+\frac{\partial P}{\partial t}+\frac{1}{2} \sigma^{2} \frac{\partial^{2} P}{\partial r^{2}}-r P+h E_{t}[P(r+J, t)-P(r, t)],^{8}
$$

with the boundary condition that $P(r, \tau=0)=1$. Given the proposed affine

solution, $P(r, \tau)=A(\tau) \exp [-B(\tau) r]$, the expectation term simplifies, and the PDDE becomes

$$
0=[a(b-r)-\lambda \sigma] \frac{\partial P}{\partial r}-\frac{\partial P}{\partial \tau}+\frac{1}{2} \sigma^{2} \frac{\partial^{2} P}{\partial r^{2}}-r P+h P(r, \tau) E_{t}[\exp [-B(\tau) J]-1],
$$

which can be expressed using the derivatives of the affine solution as two linked ODEs,

$$
\frac{\partial B}{\partial \tau}+a B=1
$$

and

$$
\frac{\frac{\partial A}{\partial \tau}}{A}=(\lambda \sigma-a b) B+\frac{1}{2} \sigma^{2} B^{2}+h E_{t}[\exp [-B(\tau) J]-1] .
$$

Assumptions regarding the distribution of jump sizes become critical at this juncture. Given the linearity of the expectations operator, the final term in the bond pricing equation (as well as of course for the ODE for $A$ ) can be written as $h P(r, \tau)\left(E_{t}\{\exp [-B(\tau) J]\}-1\right)$, and the

\footnotetext{
${ }^{6}$ This is certainly not the only way to derive the jump-augmented bond pricing equation. One could also extend the arbitrage argument in Vasicek (1977), for example.

${ }^{7}$ Merton (1976, p. 133) raises the argument that, in the case of stocks, information that precipitates jumps is likely firm or industry specific, such as "the discovery of an important new oil well or the loss of a court suit," and is therefore unlikely to move "market” prices. This argument, broadly consistent with the Capital Asset Pricing Model (CAPM), is more compelling perhaps for asset prices of individual firms as opposed to aggregate market indices or government bonds.

${ }^{8}$ Given that the equation includes an expectation, previous studies also refer to the PDDE as a partial integrodifferential equation (PIDE).
} 
expectation, $E_{t}\{\exp [-B(\tau) J]\}$, is effectively the moment generating function (m.g.f.) of the (presumed) distribution of jump sizes. For example, one common assumption (Baz and Das, 1996; Ahn and Thompson, 1988) is that $J$ is normally distributed with mean $\alpha$ and standard deviation $\gamma$, and the PDDE that incorporates this assumption given the m.g.f. of a Gaussian distribution follows

$$
0=[a(b-r)-\lambda \sigma] \frac{\partial P}{\partial r}-\frac{\partial P}{\partial \tau}+\frac{1}{2} \sigma^{2} \frac{\partial^{2} P}{\partial r^{2}}-r P+h P(r, \tau)\left(\exp \left[-\alpha B(\tau)+\frac{1}{2} \gamma^{2} B^{2}(\tau)\right]-1\right),
$$

with

$$
\frac{\partial B}{\partial \tau}+a B=1
$$

and

$$
\frac{\frac{\partial A}{\partial \tau}}{A}=(\lambda \sigma-a b) B+\frac{1}{2} \sigma^{2} B^{2}+h\left(\exp \left[-\alpha B(\tau)+\frac{1}{2} \gamma^{2} B^{2}(\tau)\right]-1\right) .
$$

The first ODE in (7) has an explicit solution, but numerical methods are necessary to solve the second. Notably, different distributional assumptions for jump sizes and therefore different m.g.f.s determine the precise form of the PDDE, the closed-form solution (if any), numerical estimate, and closed-form approximations described below.

A couple comments are noteworthy before examining linearization approximations to the bond pricing equation for jumps. First, one can easily relax the assumption that jump risk can be diversified away without considerably complicating the problem. Following Das and Foresi (1996), the pricing kernel with systematic jump risk follows

$$
\frac{d \Lambda}{\Lambda}=-r d t-\lambda d W+\lambda_{J} h d t-\lambda_{J} d \pi
$$

where the market price of (systematic) jump risk is also constant and denoted by $\lambda_{J}$. They show that the implied PDDE is 


$$
0=[a(b-r)-\lambda \sigma] \frac{\partial P}{\partial r}-\frac{\partial P}{\partial \tau}+\frac{1}{2} \sigma^{2} \frac{\partial^{2} P}{\partial r^{2}}-r P+h\left(1-\lambda_{J}\right) P(r, \tau) E_{t}[\exp [-B(\tau) J]-1] .^{9}
$$

Therefore, one can readily account for systematic jump risk in the approximations outlined below by substituting $h\left(1-\lambda_{J}\right)$ for $h^{10}$ Relaxation of the problematic assumption that jump risk is diversifiable becomes more costly in the context of estimating parameters.

Second, although the existence of closed-form solutions depends critically on the distributional assumption for $J$, one can easily solve for the Fourier transform of the bond price using the transform of the PDDE, (5). ${ }^{11}$ In short, given that differentiation and convolution both become multiplication in transform space, the PDDE simplifies to the ODE,

$$
\frac{\partial \hat{P}}{\partial \tau}+K(\xi) \hat{P}=0
$$

with the solution

$$
\hat{P}(r, \tau)=e^{-K(\xi) \tau}
$$

where

$$
K(\xi)=[a(b-r)-\lambda \sigma] i \xi+\frac{1}{2} \sigma^{2} \xi^{2}-r-h(\hat{f}(\xi)-1)
$$

$\hat{f}(\xi)$ is the characteristic function of the distribution for jumps, and $\hat{P}(r, \tau)$ denotes the Fourier transform of the bond price. ${ }^{12}$ Notably, the derivation of this solution in transform space does not depend on the presumed affine form for bond prices.

\section{The Linearization Approximation and an Alternative: Normally Distributed Jumps}

The discussion now turns to closed-form approximations of the (single-factor) bond pricing equation with jumps. After a review of existing linearization techniques examined in the

\footnotetext{
${ }^{9}$ Actually, this equation re-arranges the terms slightly compared to equation (10) in Das and Foresi (1996, p. 12).

${ }^{10}$ Sensitivity analyses along these lines similar to Tables 1-6 produces somewhat trivial differences in (approximate) bond prices and yields. Results are available on request.

${ }^{11}$ To use perhaps looser terminology, one can solve for the characteristic function of the bond price rather than the bond price itself.

${ }^{12}$ The bond price itself can be obtained by taking the inverse Fourier transform, as in$$
P(r, \tau)=\frac{1}{2 \pi} \int_{-\infty}^{\infty} e^{-i r \xi} e^{-K(\xi) \tau}
$$

But, the existence of closed-form expressions again depends on the distributional assumptions for $J$.
} 
literature, this section examines alternative approximations to the PDDE under the assumption that $J$ is normally distributed.

\subsection{Literature Review}

Ahn and Thompson (1988) and Baz and Das (1996) employ a linearization technique to produce an exact solution for bond prices with respect to an approximate PDDE. They use a two-term Taylor-series approximation of the exponential function within the expectation of equation (6), $\exp [-B(\tau) J]$, to arrive at

$$
0=[a(b-r)-\lambda \sigma] \frac{\partial P}{\partial r}-\frac{\partial P}{\partial \tau}+\frac{1}{2} \sigma^{2} \frac{\partial^{2} P}{\partial r^{2}}-r P+h P E_{t}\left[\left(1-J B+\frac{J^{2} B^{2}}{2}\right)-1\right] .
$$

This standard approximation notably does not require any explicit assumption about the distribution of jump sizes. Rather, one only needs the first and second moments of the distribution. ${ }^{13}$ Taking expectations and noting the mean and variance of $J$ by $\alpha$ and $\gamma^{2}$, respectively, the approximate PDDE is then

$$
0=[a(b-r)-\lambda \sigma] \frac{\partial P}{\partial r}-\frac{\partial P}{\partial \tau}+\frac{1}{2} \sigma^{2} \frac{\partial^{2} P}{\partial r^{2}}-r P+h P\left[-\alpha B+\frac{1}{2}\left(\mu^{2}+\gamma^{2}\right) B^{2}\right] .
$$

Given the proposed affine solution and the relevant derivatives, this equation reduces to a system of two ODEs, $B a+\frac{\partial B}{\partial \tau}=1$ and $\frac{\frac{\partial A}{\partial \tau}}{A}=(\lambda \sigma-a b-h \alpha) B+\frac{1}{2}\left[\sigma^{2}+h\left(\alpha^{2}+\gamma^{2}\right)\right] B^{2}$, and the approximate closed-form solution for bond prices given the initial data that $A(0)=1$ and $B(0)=0$ follows

$$
P(r, \tau)=\exp \left\{-\frac{1-e^{-a \tau}}{a} r+\frac{M_{1} a+M_{2}}{a^{2}} \tau+\frac{M_{1} a+2 M_{2}}{a^{3}}\left(e^{-a \tau}-1\right)-\frac{M_{2}}{2 a^{3}}\left(e^{-2 a \tau}-1\right)\right\},
$$

where

\footnotetext{
${ }^{13}$ Of course, the variance has little context without some sense of the nature of the distribution, but previous literature (Ahn and Thompson, 1988; Baz and Das, 1996) commonly assumes that $J$ is Gaussian.
} 
$M_{1}=-a b+\lambda \sigma-h \alpha$ and $M_{2}=\frac{1}{2}\left(\sigma^{2}+h\left[\alpha^{2}+\gamma^{2}\right]\right)$. Note that in order for the condition $\lim _{\tau \rightarrow \infty} P(r, \tau)=0$ to be met, we must have $M_{1} a+M_{2}<0$.

Baz and Das (1996) show that the above expression provides a very close approximation to bond prices vis-à-vis a numerical solution to the pricing equation. Again, this standard approximation does not technically require full knowledge of the distribution of $J$, but notably, in the case of a Gaussian distribution, the expectation of the two-term Taylor-series approximation of $\exp [-B(\tau) J]$ in equation (6) is not equivalent to the one-term Taylor-series approximation of the m.g.f. of a normal distribution in equation (7). That is, such an approximation would imply the (approximate) PDDE

$$
0=[a(b-r)-\lambda \sigma] \frac{\partial P}{\partial r}-\frac{\partial P}{\partial \tau}+\frac{1}{2} \sigma^{2} \frac{\partial^{2} P}{\partial r^{2}}-r P+h P\left[-\alpha B+\frac{1}{2} \gamma^{2} B^{2}\right] .
$$

However, of course (12) and (14) are equivalent if $\alpha=0$, a common assumption in the literature.

\subsection{An Alternative Closed-Form Approximation under the Gaussian Assumption}

Although the approximations in Baz and Das (1996) are very close to the numerical estimates, they suggest that "(t)he degree of accuracy of this approach is still an open question (p. 78).” To address this question further, the discussion now turns to an alternative that more explicitly incorporates the specific distributional assumptions for $J$ and employs two-term Taylor-series approximations after the expectation (the integral) in the bond pricing equation has been taken. ${ }^{14}$ Put differently, the alternative approximates the m.g.f. of the distribution for $J$. For example, given the Gaussian assumption and its m.g.f., $m_{J}(\theta)=\exp \left[-\alpha B+\frac{1}{2} B^{2} \gamma^{2}\right]$, the (approximate) PDDE follows

$$
0=[a(b-r)-\lambda \sigma] \frac{\partial P}{\partial r}-\frac{\partial P}{\partial \tau}+\frac{1}{2} \sigma^{2} \frac{\partial^{2} P}{\partial r^{2}}-r P+h P\left(-\mu B+\frac{\left(\mu^{2}+\gamma^{2}\right) B^{2}}{2}-\frac{\mu \gamma^{2} B^{3}}{2}+\frac{\gamma^{4} B^{4}}{8}\right),
$$

\footnotetext{
${ }^{14}$ Of course, one need not limit the approximations to two terms in practice.
} 
and by again making use of the initial data and the presumed affine form of the solution, the closed-form approximation for bond prices using this alternative follows

$$
\begin{aligned}
& P(r, \tau)=\exp \left\{-\frac{1-e^{-a \tau}}{a} r+\frac{M_{1} a^{3}+M_{2} a^{2}+M_{3} a+M_{4}}{a^{4}} \tau+\frac{M_{1} a^{3}+2 M_{2} a^{2}+3 M_{3} a+4 M_{4}}{a^{5}}\left(e^{-a \tau}-1\right)\right. \\
& \left.-\frac{M_{2} a^{2}+3 M_{3} a+6 M_{4}}{2 a^{5}}\left(e^{-2 a \tau}-1\right)+\frac{M_{3} a+4 M_{4}}{3 a^{5}}\left(e^{-3 a \tau}-1\right)-\frac{M_{4}}{4 a^{5}}\left(e^{-4 a \tau}-1\right)\right\}
\end{aligned}
$$

where

$M_{1}=-a b+\lambda \sigma-h \alpha, M_{2}=\frac{1}{2}\left(\sigma^{2}+h\left[\alpha^{2}+\gamma^{2}\right]\right), M_{3}=-\frac{1}{2} h \alpha \gamma^{2}$,and $M_{4}=\frac{1}{8} h \gamma^{4}$. In order for

the condition that bond prices approach zero as the time to maturity goes to infinity, i.e.

$\lim _{\tau \rightarrow \infty} P(r, \tau)=0$, the parameters must satisfy $M_{1} a^{3}+M_{2} a^{2}+M_{3} a+M_{4}<0$.

Interestingly, if $\alpha=0$, one can arrive at the same approximation in (16) using a

somewhat different approach by taking a four-term Taylor expansion of the exponential in (6) instead of the two-term expansion in Baz and Das (1996), as in

$$
0=[a(b-r)-\lambda \sigma] \frac{\partial P}{\partial r}-\frac{\partial P}{\partial \tau}+\frac{1}{2} \sigma^{2} \frac{\partial^{2} P}{\partial r^{2}}-r P+h P E_{t}\left[\left(1-J B+\frac{J^{2} B^{2}}{2}-\frac{J^{3} B^{3}}{3 !}+\frac{J^{4} B^{4}}{4 !}\right)-1\right] .
$$

Of course, in this approximation, one needs the first four moments of the distribution, but the m.g.f. is not explicitly introduced. ${ }^{15}$ Similar to the distinction between (13) and (14), the closedform approximation to (17) is not equivalent to (16) if $\alpha \neq 0$.

\subsection{Some Parameterizations using Linearization Techniques}

To address the relative accuracy of this alternative approximation, Table 1 shows bond prices and yields for integer maturity points between 0 and 30 years given a set of parameter values previously used in the literature. ${ }^{16}$ Prices and yields based on the numerical estimation, the closed-form approximations following Ahn and Thompson (1988) and Baz and Das (1996), and the proposed alternative closed-form approximations are listed in Columns 2 and 3, Columns

\footnotetext{
15 Then again, one is likely to use the m.g.f., if available, to derive higher moments.

16 The parameter values follow those in Exhibit 5 from Baz and Das (1996, p. 83). The numerical approximations use the NDSolve command in Mathematica, which follow a modified Runge-Kutta procedure.
} 
4 and 5, and Columns 7 and 8, respectively. Column 6 reports the absolute value of the difference in basis points between the yields implied by the standard linearization approximation vis-à-vis those implied by the numerical solution. The deviations for this set of parameters average about 0.182 basis points for each integer maturity point between one and 30 years, and the accuracy seems to wane as maturity lengthens. Column 9 reports the same results for the alternative closed-form approximation, (16), and indicates that the average absolute deviation is about 0.000018 basis points, notably lower than the standard technique, but the accuracy again appears to increase somewhat out the term structure. With respect to comparing the two closedform approximations, as Column 10 indicates, on average the alternative approximation is about 2737 times as accurate as the standard linearization technique across each maturity point. Also, Column 10 indicates that the improvement is largely uniform, although the standard approximation is curiously more accurate with one year to maturity. But in general, this particular analysis suggests that the alternative closed-form approximation is quite close to the numerical approximation.

Of course, Table 1 does not provide definitive evidence regarding the accuracy of either closed-form approximation, because the choice of parameters is ultimately arbitrary. Again, the parameters in Table 1 are consistent with common assumptions made in previous literature to facilitate comparisons. ${ }^{17}$ Consideration of the relevant universe of alternatives in this context is not feasible, but perhaps those parameters related to the variance of the short rate process are of particular interest, specifically the relative contribution to total variance from the diffusion parameter, $\sigma$, on the one hand, and the contribution of the jump parameter(s) $-\alpha, \gamma$, and $h$ if $J$ is normally distributed - on the other. In short, given that the approximations only involve those terms in the PDDE related to jumps, one might expect the closed-form solution to the estimated PDDE to be more accurate the smaller the contribution of jumps to total variance of the short rate process. To measure the relative contributions, note that if jumps are normally distributed, the unconditional variance of the jump-diffusion short rate process in (1) follows

\footnotetext{
${ }^{17}$ To address the sensitivity of parameter selection in the standard linearization technique, Baz and Das (1996) sequentially vary each of the parameters.
} 


$$
\frac{\sigma^{2}+h\left(\alpha^{2}+\gamma^{2}\right)}{2 a} .^{18}
$$

Therefore, the ratio $\sigma^{2} / h\left(\alpha^{2}+\gamma^{2}\right)$ provides a non-dimensional measure of the relative contributions, and the value of (18) given the chosen parameters in Table 1 indicates that the contribution of diffusion is 6.4 times that of the jumps. ${ }^{19}$ Although the jump component in this case is not negligible, perhaps an alternative parameterization that increases its relative contribution would be instructive.

To that end, Table 2 shows the results from a parameterization in which the jump contribution is twice that of the diffusion, ${ }^{20}$ with $\sigma=.02, h=16$, and the remaining parameter values set to those in Table $1 .^{21}$ The results are similar to Table 1 , as the alternative closed-form approximation is more accurate than the standard linearization technique, as indicated in Column 10. Also, the differences between the approximations and the numerical solutions, as shown in Columns 6 and 9, are somewhat larger than the corresponding differences in Table 1. Even so, the magnitudes of the differences still suggest a high degree of accuracy - the alternative closedform approximation is never even as much as a thousandth of a basis point from the numerical estimate across the term structure. Therefore, at least given this set of parameters, the closedform approximations seem quite close to the numerical solutions, even when jumps contribute more than diffusion to the variance in the short rate.

\section{Alternative Distributional Assumptions for Jump Sizes}

The discussion now turns to two alternative assumptions for the distribution of jump sizes. The first follows Das and Foresi (1996), who assume that the absolute value of jump size follows an exponential distribution, ${ }^{22}$ with the mean absolute jump size denoted by $\alpha^{-1}$, and the jump sign follows a Bernoulli distribution, with the probability of a positive jump denoted by $w$.

${ }^{18}$ More generally, the unconditional variance of the short rate process is $\frac{\sigma^{2}+h E_{t}\left[J^{2}\right]}{2 a}$. See the discussion of GMM in Section 5 for a derivation.

${ }^{19}$ The diffusive and jump contributions are of course equal when $\sigma=\sqrt{h} \gamma$.

${ }^{20}$ Johannes (2004) finds that jumps account for more than half (almost two-thirds) of the condition variance of interest rate changes at low (high) levels.

${ }^{21}$ The condition $M_{1} a^{3}+M_{2} a^{2}+M_{3} a+M_{4}<0$ still holds for this parameterization.

${ }^{22}$ Johannes (2004, pp. 242-243) assumes that jumps follow a log-normal distribution. 
Notably, as Das and Foresi (1996) show, this assumption produces a closed-form solution for bond prices. Therefore, in contrast to Baz and Das (1996), one can adopt this distributional assumption and directly assess the relative accuracy of the numerical and closed-form approximations with respect to an analytical solution. In other words, one can test whether numerical solutions to the exact PDDE are more accurate than closed-form solutions to an approximation PDDE.

The second distribution assumption, which the existing literature on jump-diffusion processes and ATSMs does not consider, directly addresses the prospect that, in most notable contract to the Gaussian case, jump sizes are bi-modally distributed with modes sufficiently distant from the origin. A Bernoulli mixture of two normal distributions fits this description, and although this distributional assumption for $J$ does not produce a closed-form solution, this section compares the accuracy of two alternative closed-form approximations vis-à-vis the numerical solution, similar to the analysis in the previous section.

\subsection{The Exponential Distributional Assumption for J}

Rather than theoretical notions about the true distribution of jump sizes, consideration of the alternative distribution from Das and Foresi (1996) is driven more by the fact that it produces an analytical solution for bond prices which, in turn, allows us to compare the accuracy of numerical solutions with closed-form solutions to the approximate PDDE. Given the m.g.f. of an exponential distribution and the probability of a positive jump, ${ }^{23} \mathrm{w}$, we can derive the moments necessary to calculate both the standard linearization technique as well as a version of the alternative closed-form alternative in the previous section. Given this information, the bond pricing equation using the latter approximation is

${ }^{23}$ The m.g.f., $m_{J}(\theta)$, of an exponential distribution follows $m_{J}(\theta)=\frac{\alpha}{\alpha-\theta}$, and the $n^{\text {th }}$ moment of the distribution follows $\left.\frac{\partial^{(n)}}{\partial t} m(t)\right|_{t=0}=\frac{n !}{\alpha^{n}}$. Therefore, following the distribution in Das and Foresi (1996), the expected value of jumps follows $w \frac{1}{\alpha}+(1-w)\left(-\frac{1}{\alpha}\right)$. 


$$
0=[a(b-r)-\lambda \sigma] \frac{\partial P}{\partial r}-\frac{\partial P}{\partial \tau}+\frac{1}{2} \sigma^{2} \frac{\partial^{2} P}{\partial r^{2}}-r P+h P\left(-\frac{(2 w-1) B}{\alpha}+\frac{B^{2}}{\alpha^{2}}-\frac{(2 w-1) B^{3}}{\alpha^{3}}+\frac{B^{4}}{\alpha^{4}}\right),{ }^{24}
$$

and the solution follows (16) (with the same restrictions) but with $M_{1}=-a b+\lambda \sigma-h \frac{(2 w-1)}{\alpha}$, $M_{2}=\frac{\sigma^{2}}{2}+\frac{h}{\alpha^{2}}, M_{3}=-h \frac{(2 w-1)}{\alpha^{3}}$, and $M_{4}=\frac{h}{\alpha^{4}}$. Again, the standard linearization only considers the first two moments of the distribution, and the solution also follows (16) but with $M_{1}=-a b+\lambda \sigma-h \frac{(2 w-1)}{\alpha}, M_{2}=\frac{\sigma^{2}}{2}+\frac{h}{\alpha^{2}}, M_{3}=0$, and $M_{4}=0$.

Table 3 examines the accuracy of the numerical solution and the two alternative closedform approximations. Columns 2 and 3 list the closed-form solution from Das and Foresi (1996, pp. 12-13) in terms of bond prices and yields, respectively, given an arbitrary but reasonable set of parameters that match the assumptions in Table 1 and the parameterization in Das and Foresi (1996, p. 15) as closely as possible for all integer maturity points between 0 and 30 years. Columns 4 and 5, Columns 7 and 8, and Columns 10 and 11 list prices and yields from the corresponding numerical solution, standard linearization technique, and the alternative closedform approximation, respectively. Column 6 indicates the absolute difference in yields between the closed-form solution and the numerical estimates expressed in basis points. On average from one to 30 years to maturity, the numerical approximation is within about .000069 basis points of the actual solution. Column 9 lists the corresponding differences using the standard linearization technique, which on average is about .091 basis points across the maturity spectrum. Also, Column 12 lists the absolute difference in yields between the alternative closed-form approximation and the actual solution, which averages about .00014 basis points from one to 30 years to maturity. Therefore, in general, the absolute accuracy of both closed-form approximations seems favorable using an alternative distributional assumption for $J$. Moreover, with respect to the relative accuracy of the methods, Column 13 shows that on average the alternative closed-form approximation is actually more precise than the numerical solution. This result is notably driven by greater accuracy from one to 10 years to maturity, after which the numerical approximation is superior, but this result generally questions the use of numerical solutions as a benchmark for precision. Also, as indicted in Column 14 and consistent with

${ }^{24}$ This approximation broadly follows (17). 
Tables 1 and 2, the alternative is clearly more accurate than the standard linearization technique on average and for each maturity point.

With respect to an alternative parameter set, consider again the relative contribution of the diffusion and jump volatility parameters to the total variance of the short rate. In the case of the exponential distribution, the unconditional variance follows

$$
\frac{\sigma^{2}+h\left(\frac{2}{\alpha^{2}}\right)}{2 a},
$$

and given the set of parameters in Table 3, the diffusion contribution is 12.8 times that of the jumps. Similar to the Gaussian assumption discussed previously, the case in which the jump contribution is twice that of the diffusion component - which again obtains with $\sigma=.02, h=$ 16, and holding $\gamma$ constant - is perhaps instructive. As Columns 9 and 12 in Table 4 suggest, the closed-form approximations are somewhat less accurate compared to the corresponding estimates in Table 3. Nonetheless, the alternative approximation is never even as much as seventy thousandths of a basis point from the closed-form solution, and as Column 13 shows, the estimates are on average closer than the numerical solution, albeit not for those at five and after 10 years to maturity.

Therefore, even when jumps contribute twice as much as diffusion to variance in the short rate, the alternative closed-form approximation produces very accurate estimates under certain reasonable cases, and its comparative precision suggests that use of the numerical solution as an absolute benchmark is potentially questionable. That is, one cannot in principle be sure whether approximate solutions to the exact PDDE produce more precise estimates than exact solutions to an approximate PDDE. ${ }^{25}$

\subsection{The Bi-modal Gaussian Mixture Distributional Assumption for $\mathrm{J}$}

So far, the discussion of alternative distributional assumptions for jump sizes has not appealed to much intuition about the true form of the density. In fact, the common assumption in the literature explored in Tables 1 and 2 that $J$ has an expected value of zero and follows a normal distribution seems somewhat problematic. Simply put, if $J$ is both centered around the

\footnotetext{
${ }^{25}$ More generally, higher-order Taylor expansions would result in approximate PDDEs that would of course be more accurate than the two-term approximations used in the alternative linearization technique.
} 
origin and Gaussian, the most common jump (i.e. the mode) would not seem to be a jump (i.e. a non-local change) in any meaningful sense of the word. Rather, the assumption that the average jump size is zero only seems tenable if jump size is bi-modally distributed, as (large) positive and negative values cancel when taking the average (or forming expectations).

The assumption in Das and Foresi (1996) that the absolute value of jump size follows an exponential distribution and that jump sign follows a Bernoulli distribution is a considerable improvement in this regard, with the component means at $-\alpha^{-1}$ and $\alpha^{-1}$ under the assumption that $w=0.5$. However, another desirable feature of any possible distribution for jump sizes would seem to be that the modes are of considerable distance from the origin, given the fundamental motivation to incorporate the possibility of sizeable asset price movements. In addition, jumps might also be symmetrically distributed around the means, which is not a feature of exponential distributions.

Therefore, a useful alternative distribution would be bi-modal with jumps symmetrically distributed around modes of sufficient distance from the origin. ${ }^{26}$ To arrive at such a density, consider a general Gaussian mixture, as in

$$
f(J)=\sum_{i=1}^{n} w_{i} \frac{1}{\sqrt{2 \pi \gamma_{i}^{2}}} \exp \left[-\frac{\left(J-\alpha_{i}\right)^{2}}{2 \gamma_{i}^{2}}\right]
$$

where $f(J)$ is the total density of the mixture, $w_{i}$ is the weight assigned to the $i^{\text {th }}$ component Gaussian distribution, $n$ is the number of component distributions in the mixture, $\alpha_{i}$ is the mean of the $i^{\text {th }}$ component distribution, and $\gamma_{i}^{2}$ is the variance of the $i^{\text {th }}$ component distribution. For this specific application, $n=2$, which results in

$$
f(J)=\frac{w}{\sqrt{2 \pi} \gamma_{1}} \exp \left[-\frac{\left(J-\alpha_{1}\right)^{2}}{2 \gamma_{1}^{2}}\right]+\frac{(1-w)}{2 \sqrt{2 \pi} \gamma_{2}} \exp \left[-\frac{\left(J-\alpha_{2}\right)^{2}}{2 \gamma_{2}^{2}}\right] .
$$

Also, the moment generating function for this distribution, $m_{J}(\theta)$, follows

\footnotetext{
${ }^{26}$ Insofar as the short rate is a policy rate, such as the federal funds rate in the U.S., one could also make the case that jump sizes follow a discrete multinomial distribution. Such a distribution can also be bi-modal, with the modes sufficiently far from the origin. However, a pure jump process, as opposed to a jump-diffusion, might better characterize the target funds rate process. See Piazzesi (2005) for an analysis of jumps and the target federal funds rate.
} 


$$
m_{J}(\theta)=w \exp \left[\mu_{1} \theta+\frac{1}{2} \theta^{2} \gamma_{1}^{2}\right]+(1-w) \exp \left[\mu_{2} \theta+\frac{1}{2} \theta^{2} \gamma_{2}^{2}\right]
$$

Returning to the fundamental PDDE of bond prices for jump-diffusions and remembering that the expectation resembles the m.g.f., where $-B(\tau)$ is the constant, the PDDE in the case of the Gaussian mixture becomes

$$
\begin{aligned}
0= & {[a(b-r)-\lambda \sigma] \frac{\partial P}{\partial r}-\frac{\partial P}{\partial \tau}+\frac{1}{2} \sigma^{2} \frac{\partial^{2} P}{\partial r^{2}}-r P } \\
& +h P(r, \tau)\left[w \exp \left[-\mu_{1} B+\frac{1}{2} B^{2} \gamma_{1}^{2}\right]+(1-w) \exp \left[-\mu_{2} B+\frac{1}{2} B^{2} \gamma_{2}^{2}\right]-1\right]
\end{aligned}
$$

which can only be solved numerically. ${ }^{27}$

Turning to the approximations of the PDDE, recall that the standard linearization technique requires only the first and second moments of $J$, and therefore the PDDE follows

$$
\begin{aligned}
0= & {[a(b-r)-\lambda \sigma] \frac{\partial P}{\partial r}-\frac{\partial P}{\partial \tau}+\frac{1}{2} \sigma^{2} \frac{\partial^{2} P}{\partial r^{2}}-r P } \\
& +h P(r, \tau)\left[-\left[w \alpha_{1}+(1-w) \alpha_{2}\right] B+\frac{\left[w\left(\alpha_{1}^{2}+\gamma_{1}^{2}\right)+(1-w)\left(\alpha_{2}^{2}+\gamma_{2}^{2}\right)\right]}{2} B^{2}\right]
\end{aligned}
$$

The solution to this equation again follows (16) but with $M_{1}=-a b+\lambda \sigma-h\left[w \alpha_{1}+(1-w) \alpha_{2}\right]$, $M_{2}=\frac{1}{2}\left(\sigma^{2}+h\left[w\left(\alpha_{1}^{2}+\gamma_{1}^{2}\right)+(1-w)\left(\alpha_{2}^{2}+\gamma_{2}^{2}\right)\right]\right), M_{3}=0$, and $M_{4}=0 .{ }^{28}$ With respect to the corresponding alternative approximation that considers the two-term Taylor series approximation of the m.g.f. of the distribution, (24), the approximate PDDE follows,

\footnotetext{
${ }^{27}$ One could entertain the possibility that the weight between the two distributions is functionally dependent on the distance between $r$ and $b$. That is, negative (positive) jumps might be more likely if $r$ is sufficiently above (below) the mean.

${ }^{28}$ Notably, one does not obtain the same result if one takes the one-term Taylor series approximation of the m.g.f. of the Bernoulli mixture of Gaussians. In this case, $M_{2}=\frac{1}{2}\left(\sigma^{2}+h\left[w \gamma_{1}^{2}+(1-w) \gamma_{2}^{2}\right]\right)$.
} 


$$
\begin{aligned}
0= & {[a(b-r)-\lambda \sigma] \frac{\partial P}{\partial r}-\frac{\partial P}{\partial \tau}+\frac{1}{2} \sigma^{2} \frac{\partial^{2} P}{\partial r^{2}}-r P } \\
& +h P(r, \tau)\left\{-\left[w \alpha_{1}+(1-w) \alpha_{2}\right] B+\frac{1}{2}\left[w\left(\gamma_{1}^{2}+\alpha_{1}^{2}\right)+(1-w)\left(\gamma_{2}^{2}+\alpha_{2}^{2}\right)\right] B^{2}\right. \\
& \left.-\frac{1}{2}\left[w \alpha_{1} \gamma_{1}^{2}+(1-w) \alpha_{2} \gamma_{2}^{2}\right] B^{3}+\frac{1}{8}\left[w \gamma_{1}^{4}+(1-w) \gamma_{2}^{4}\right] B^{4}\right\}
\end{aligned}
$$

where the solution again follows (16) but with $M_{1}=-a b+\lambda \sigma-h\left[w \alpha_{1}+(1-w) \alpha_{2}\right]$,

$$
\begin{aligned}
& M_{2}=\frac{1}{2}\left(\sigma^{2}+h\left[w\left(\gamma_{1}^{2}+\alpha_{1}^{2}\right)+(1-w)\left(\gamma_{2}^{2}+\alpha_{2}^{2}\right)\right]\right), M_{3}=-\frac{1}{2} h\left[w \alpha_{1} \gamma_{1}^{2}+(1-w) \alpha_{2} \gamma_{2}^{2}\right], \text { and } \\
& M_{4}=\frac{1}{8} h\left[w \gamma_{1}^{4}+(1-w) \gamma_{2}^{4}\right] .
\end{aligned}
$$

Similar to Table 1, Table 5 shows prices and yields based on the numerical estimation, the standard linearization technique, and the alternative closed-form approximation in Columns 2 and 3, Columns 4 and 5, and Columns 7 and 8, respectively. The chosen parameters follow those in Tables 1 and 3 as closely as possible; the component distributions of the Gaussian mixtures have means of 60 and -40 basis points, with standard deviations of 15 and 10 basis points, respectively; and the probability of a positive jump is 40 percent. As Column 6 indicates, the average absolute difference across the maturity spectrum between the numerical solution and the standard linearization approximation is about 0.18 basis points, and the precision seems to decrease with maturity. Also, Column 9 indicates that the alternative closed-form approximation is on average within about 0.15 basis points of the numerical solution, and Column 10 indicates that the alternative is consistently more precise than the standard method out the term structure but, at lease given this set of parameters, does not provide much greater accuracy. ${ }^{29}$

With respect to alternative parameterizations, the unconditional variance of the short-rate process under the Gaussian mixture assumption follows

$$
\frac{\sigma^{2}+h\left[w\left(\alpha_{1}^{2}+\gamma_{1}^{2}\right)+(1-w)\left(\alpha_{2}^{2}+\gamma_{2}^{2}\right)\right]}{2 a},
$$

and the relative contribution of diffusion is just over 25 times that of jumps given the parameter values in Table 5. Table 6 considers an alternative parameterization in which jumps contribute about twice as much as the diffusive component to total variance, with $\sigma=.02, h=31$, and the

\footnotetext{
${ }^{29}$ Again, given no closed-form solution, one ultimately cannot judge the accuracy of the numerical approximation.
} 
remaining parameters as in Table $5 .^{30}$ Similar to Tables 2 and 4, the results generally suggest that both closed-form approximations become somewhat less accurate the more jumps contribute to total variance in the short rate. For example, Column 9 indicates that the alternative closedform approximation is on average within about 0.466 basis points of the numerical solution across the maturity spectrum and just over a basis point at 30 years. However, the absolute degree of precision is roughly comparable.

\subsection{A “Restricted” Bi-modal Mixture of Gaussians}

A few simplifying assumptions regarding the Gaussian mixture seem intuitive. That is, perhaps the component distributions of $J$ are simply symmetric reflections across the origin, with equal but opposite means and equal variances. Under this formulation, $\alpha_{2}=-\alpha_{1}, \gamma_{2}=\gamma_{1}$, and $w$ $=0.5$, and the PDDE using the alternative closed-form approximation becomes

$$
0=[a(b-r)-\lambda \sigma] \frac{\partial P}{\partial r}-\frac{\partial P}{\partial \tau}+\frac{1}{2} \sigma^{2} \frac{\partial^{2} P}{\partial r^{2}}-r P+h P(r, \tau)\left\{\frac{1}{2}\left[\left(\gamma_{1}^{2}+\alpha_{1}^{2}\right)\right] B^{2}+\frac{1}{8} \gamma_{1}^{4} B^{4}\right\} .
$$

The solution again follows (16) but with $M_{1}=-a b+\lambda \sigma, M_{2}=\frac{1}{2}\left(\sigma^{2}+h\left[\gamma_{1}^{2}+\alpha_{1}^{2}\right]\right)$, $M_{3}=0$, and $M_{4}=\frac{1}{8} h \gamma_{1}^{4}$. Interestingly, the bi-modal Gaussian mixture implies an approximate PDDE that is no more complex than that under the assumption that $J$ is normally distributed (and uni-modal). Also, this “restricted” Gaussian mixture requires three fewer parameters to estimate than the full model and the same number as the model in which $J$ is Gaussian and uni-modal.

\subsection{How Critical is the Distributional Assumption for Jumps?}

How sensitive are estimates for bond yields to the distributional assumption for jumps? More specifically, what is the cost in accuracy of assuming that jumps are uni-modal and normally distributed if the "true" distribution is, say, a bi-modal mixture exponentials or Gaussians? One imperfect way to address this issue is to assume a constant mean and variance for jump size but alter the specific form of the distribution and compare the corresponding

\footnotetext{
${ }^{30}$ The precise ratio of the diffusive to jump contributions to total variance given the parameterization in Table 5 (Table 6) is 25.098 (0.506009).
} 
closed-form approximations for yields derived from the two-term Taylor series approximations of the m.g.f. in the bond pricing equation. Toward that end, Column 2 in Table 7 shows the term structure of interest rates out to 30 years given the parameter assumptions in Table 1, which again stipulate that jumps are normally distributed with a zero mean and a standard deviation of 100 basis points. $^{31}$ Alternatively, Column 3 shows the results with the same mean and variance as in Column 2, but jumps follow the exponential distribution described in Das and Foresi (1996). ${ }^{32}$ Similarly, Column 4 shows the term structure assuming jumps follow a bi-modal Bernoulli mixture of Gaussians, ${ }^{33}$ and Column 5 follows its "restricted" form where $\alpha_{2}=-\alpha_{1}$, $\gamma_{2}=\gamma_{1}$, and $w=0.5 .^{34}$

Given this particular set of parameters and holding constant the mean and variance for jumps, the implied term structure of interest rates displays marginal differences across distributional assumptions for $J$. For example, the average difference across each maturity between the uni-modal Gaussian and the exponential, Gaussian mixture, and "restricted" Gaussian mixture is only about $0.18,0.28$, and 0.08 basis points, respectively, as shown in Columns 6, 7, and 8 . Table 8 considers an alternative set of parameters where the intensity of jumps increases fivefold, with $h=50$, but the standard deviation of jumps is 50 basis points, half the size assumed in Table $7 .{ }^{35}$ With average absolute differences of about $0.06,0.18$, and 0.06 basis points across the term structure for the three distributional assumptions, Columns 6, 7, and 8 again suggest that alternative distributional assumptions do not seem to have a pronounced affect on yields using the alternative closed-form approximation, at least for this parameterization.

\footnotetext{
${ }^{31}$ Therefore, the estimates match Column 8 in Table 1.

${ }^{32}$ In order for jumps to have a standard deviation of 100 basis points for the exponential distribution, we must have $\alpha=141.421$.

${ }^{33}$ For the Bernoulli mixture of Gaussians, the parameters follow those in Table 5, but in order for the distribution to match the variance of the uni-modal Gaussian assumption, we must have $\gamma_{1}=0.00901436$ and $\gamma_{2}=0.00851436$. (The difference in standard deviations of the two component distributions is fixed at five basis points.) Notably, given means of 60 and -40 basis points, standard deviations of about 90.1 and 85.1 basis points, respectively, seem excessive in that the component distributions for both positive and negative jumps have considerable mass on the opposite side of the origin.

${ }^{34}$ In order for jumps to have a standard deviation of 100 basis points for the "restricted" bi-modal Bernoulli mixture of Gaussians, we must have $\gamma_{1}=0.00866025$.

${ }^{35}$ Lowering the stipulated value of the variance of $J$ to 50 basis points produces considerably more intuitive assumptions for the Bernoulli mixture of Gaussians, as $\gamma_{1}=0.00126954$ and $\gamma_{2}=0.000769536$. Therefore, there is negligible mass on the opposite side of the origin for the component distributions for positive and negative jumps. (However, to meet the restriction in the case of the "restricted" Bernoulli mixture of Gaussians, we must have $\gamma_{1}=$ 0.) Also, for the exponential distributions, $\alpha=282.843$.
} 
One should not necessarily make strong inferences about the relative importance of distributional assumptions for $J$ from this imperfect exercise. In particular, while one must hold the first and second moments constant across distributional assumptions to facilitate comparisons, variance has less meaning without the full context of the distribution. For example, the assumption that the variance of jumps is 100 basis points, the stipulation in Table 1, may seem sensible in the context of a uni-modal normal distribution centered around the origin, but, say, a Bernoulli mixture of Gaussians with the same (overall) variance might not have much intuitive appeal. Moreover, there is no substantive reason to knowingly use the "wrong” distribution. Perhaps consideration of actual data would be instructive in this regard, and indeed the discussion now turns to parameter estimation.

\section{Parameter Estimation}

Although there is a burgeoning literature on parameter estimation methods for ATSMs in which the underlying state variables follow pure diffusion processes, comparatively few studies address the case of jump-diffusion (Das, 2002, p. 34). This section considers three general methods to estimate a single-factor model of the term structure of United States Treasury yields using data from January 4, 1988 through October 12, 2005. Although previous studies have produced mixed results, the analysis first considers the general method of moments (GMM) and then turns to two applications of maximum likelihood estimation (MLE). Both MLE applications use a discrete analogue to the continuous short-rate process. But, one relies on direct proxies for the short rate, and another exploits the closed-form approximations for bond prices (and yields) to transform the approximate density similar to Pearson and Sun (1994).

\section{1. $G M M$}

Parameter estimation of the model using a variant of the GMM is as follows. The backward Kolmogorov equation implied by the jump-augmented stochastic process for the short rate, (1), can be solved to obtain the conditional characteristic function (c.c.f.). In turn, one can derive (conditional and unconditional) moments of the distribution from the c.c.f., expressed as a function of the moments of $J$ and other model parameters. Given a specific distributional assumption and a corresponding m.g.f. for $J$, one can then match the (conditional and/or 
unconditional) moments from the c.c.f. with corresponding (conditional and/or unconditional) sample moments to determine the model parameters.

To derive the c.c.f. and the moments of the distribution, consider again expression (1). Following the general approach in Heston (1993), Bates (1993), Das and Foresi (1996), and Das (2002), assume that the current time is $t=0$ and that one is interested in the distribution of $r(\tau)$ at time $t=\tau$, given the current value of the interest rate $r(0)=r$. To derive the $\tau$-interval characteristic function for the process, $\phi(r, \tau ; \xi)$, one can solve the implied backward Kolmogorov equation, which is twice-differentiable in the interest rate, is once-differentiable in $\tau$, has initial data $\phi(r, 0 ; \xi)=\exp (i \xi r)$ with arbitrary parameter $\xi$, and follows

$$
0=-\frac{\partial \phi}{\partial \tau}+[a(b-r)] \frac{\partial \phi}{\partial r}+\frac{1}{2} \sigma^{2} \frac{\partial^{2} \phi}{\partial r^{2}}+h E_{t}[\phi(r+J, t)-\phi(r, t)]
$$

The conjectured (affine) solution to the above equation is

$$
\phi(r, \tau ; \xi)=\exp [\hat{A}(\tau ; \xi)-r \hat{B}(\tau ; \xi)]
$$

where

$$
\hat{B}(\tau ; \xi)=-i \xi e^{-a \tau}
$$

and

$$
\hat{A}(\tau ; \xi)=\int i a b \xi e^{-a \tau}-\frac{1}{2} \sigma^{2} \xi^{2} e^{-2 a \tau}+h E_{t}\left[e^{i \xi e^{-a \tau} J}-1\right] d \tau
$$

In general, one can derive the $n^{\text {th }}$ conditional moment, $\mu_{c, n}$, of the distribution for the interest rate from the c.c.f. following

$$
\mu_{c, n}=\left.i^{-n} \frac{\partial^{n} \phi}{\partial \xi^{n}}\right|_{\xi=0}
$$


Furthermore, ${ }^{36}$ under the (unproblematic) restriction that $a>0$, the first four unconditional moments, $\mu_{n}$, which follow $\mu_{n}=\lim _{\tau \rightarrow \infty} \mu_{c, n}$, are

$$
\begin{aligned}
& \mu_{1}=b+\frac{h E_{t}[J]}{a}, \\
& \mu_{2}=\mu_{1}^{2}+\frac{\sigma^{2}+h E_{t}\left[J^{2}\right]}{2 a}, \\
& \mu_{3}=\mu_{1}^{3}+\frac{3 \mu_{1}\left(\sigma^{2}+h E_{t}\left[J^{2}\right]\right)}{2 a}+\frac{h E_{t}\left[J^{3}\right]}{3 a}, \\
& \mu_{4}=\mu_{1}^{4}+\frac{6 \mu_{1}^{2}\left(\sigma^{2}+h E_{t}\left[J^{2}\right]\right)}{2 a}+\frac{4 \mu_{1} h E_{t}\left[J^{3}\right]}{3 a}+\frac{3\left(\sigma^{2}+h E_{t}\left[J^{2}\right]\right)^{2}}{4 a^{2}}+\frac{h E_{t}\left[J^{4}\right]}{4 a}
\end{aligned}
$$

Note that these are the exact unconditional moments of the short rate process with no approximations. Distributional assumptions regarding $J$ are required to proceed further, and one can determine the $E_{t}\left[J^{n}\right]$ in (32) from the relevant m.g.f. (In the case of the bi-modal Gaussian distribution, under the physical measure, the model requires estimates of nine parameters, including three associated with the drift and diffusion of the process $-a, b$, and $\sigma-$ and six from the jump component $-h, w, \alpha_{1}, \alpha_{2}, \gamma_{1}, \gamma_{2}$.)

Given specification of the moments from the characteristic function, the (unconditional) sample moments follow

$$
\hat{\mu}_{n}=\frac{1}{N} \sum_{i=1}^{N} r^{n}
$$

where $N$ is the number of observations, and $r$ is the proxy for the interest rate observed at some frequency. Consider the difference between (32) and (33) for an arbitrary number of unconditional moments, as in

\footnotetext{
${ }^{36}$ Similarly, Das (2002) derives the c.c.f. and the corresponding conditional (rather than unconditional) moments with respect to the physical measure.
} 


$$
f=\left[\begin{array}{c}
f_{1} \\
f_{2} \\
\vdots \\
f_{n}
\end{array}\right]=\left[\begin{array}{c}
\mu_{1}-\hat{\mu}_{1} \\
\mu_{2}-\hat{\mu}_{2} \\
\vdots \\
\mu_{n}-\hat{\mu}_{n}
\end{array}\right] .
$$

Denoting the set of parameters by $\Psi$, the GMM estimator is

$$
\hat{\Psi}=\arg \min _{\hat{\Psi}} \Upsilon
$$

with

$$
\Upsilon=f^{\prime} W f,
$$

where $W$ is some weighting matrix, ${ }^{37}$ and $\hat{\Psi}=\left(\hat{a}, \hat{b}, \hat{\sigma}, \hat{h}, \hat{w}, \hat{\alpha}_{1}, \hat{\alpha}_{2}, \hat{\gamma}_{1}, \hat{\gamma}_{2}\right)$ if $J$ follows a Bernoulli

Gaussian mixture. The standard errors for the $i^{\text {th }}$ parameter in $\Psi$ follow $\sqrt{\Sigma_{i i}}$, where

$$
\Sigma=\frac{\partial f}{\partial \Psi} ' W \frac{\partial f}{\partial \Psi} .
$$

\subsection{GMM Results}

Given that estimation biases potentially result from the choice for the short rate proxy (Chapman et al., 1999), the analyses use three alternatives using daily data over the period from January 4, 1988 through October 12, 2005 - the overnight federal funds rate, following Das (2002), as well as one- and three-month U.S. Treasury bill rates also common in the literature. ${ }^{38}$ The GMM estimations also examine five alternative assumptions regarding jumps, including a base model without jumps (the pure Gaussian Vasicek model) and jump-diffusion models where $J$ alternatively follows a uni-modal Gaussian, a Bernoulli Gaussian mixture and its "restricted" form, and a bi-modal mixture of uniform distributions. The estimates are under the physical measure, following previous literature (Das, 1998), and use the first ten moments of the shortrate process.

Table 9 includes estimates for the Vasicek model as well as the model that assumes jumps are normally distributed. With respect to the former, as Columns 1-3 indicate, each estimate of $a$ and $b$ - the mean reversion and central tendency parameters, respectively - is positive,

\footnotetext{
${ }^{37} \mathrm{~W}$ is usually defined by the inverse of the variance-covariance matrix of the sample moments.

${ }^{38}$ With respect to the controversy over proxies for the short rate, Hamilton (1996) notes that microstructure effects such as settlement Wednesdays and quarter-ends induce jumps in daily federal funds rate series.
} 
statistically significant, and very generally consistent with findings in previous literature across the three short rate proxies. The estimates for the diffusion parameter, $\sigma$, are positive and of the generally expected magnitude, but they are statistically insignificant from zero in each case. Turning to the latter model with jumps that are uni-modal and normally distributed, the GMM estimates in Columns 4 and 6 using the federal funds rate and the three-month bill yield as proxies suggest that $a, b$, and $h$ are positive and statistically significant. The remaining estimates are of plausible magnitude but are not statistically significant. Also, as Column 5 indicates, only the estimate of $h$ is statistically significant using the one-month bill as the proxy for the short rate. Notably perhaps, the three proxies produce a range of estimates for jump intensity.

Table 10 presents the results for models that assume jumps follow a Bernoulli mixture of Gaussians, and Columns 1-2 suggest that $a$ is positive, statistically significant, and of a magnitude that is generally consistent with intuition. In addition, $h$ is statistically significant using each proxy, but the estimates of 68.02 and 76.48 jumps per year using the federal funds rate and the three-month bill yield as short rate proxies seem excessive. None of the other parameters are statistically significant. Columns 4-6 present the results from the "restricted" model for the mixture of Gaussians. The estimates of $b$ and $h$ are positive, statistically significant, generally consistent with intuition, and quite similar in magnitude across each proxy for the short rate, but none of the other estimates, except $b$ in Column 10, are statistically significant.

Finally, Table 11 presents the results under the assumption that $J$ follows a Bernoulli mixture of uniform distributions. ${ }^{39}$ Jump intensity is statistically significant and positive using each of the three short rate proxies. However, the estimates of 103.51 and 163.10 jumps per year in Columns 1 and 3, respectively, are incredible. Also, the estimate for $w$ using the federal funds rate proxy is perversely negative and statistically significant, and none of the other estimates, expect that for $a$ using the three-month bill proxy, are statistically significant.

${ }^{39}$ Given the m.g.f. of the mixture of uniform distributions, the approximate bond pricing equation becomes

$$
0=[a(b-r)-\lambda \sigma] \frac{\partial P}{\partial r}-\frac{\partial P}{\partial \tau}+\frac{1}{2} \sigma^{2} \frac{\partial^{2} P}{\partial r^{2}}-r P+\frac{1}{2} h P\left[w\left(\omega_{1}+v_{1}\right)+(1-w)\left(\omega_{2}+v_{2}\right) B\right],
$$

and the approximate closed-form solution again follows (16) but with $M_{1}=-a b+\lambda \sigma-h\left[\frac{1}{2} w\left(\omega_{1}+v_{1}\right)+\frac{1}{2}(1-w)\left(\omega_{2}+v_{2}\right)\right], M_{2}=\frac{1}{2} \sigma^{2}, M_{3}=0$, and $M_{4}=0$. 
In short, considering the dearth of statistically significant estimates and a few perverse findings, the GMM results are not very promising. ${ }^{40}$ Although the statistical significance of $h$ in every specification broadly suggests evidence for jumps, the implied intensities vary widely across short rate proxies and distributional assumptions for $J$. Notably, very broadly related estimations based on the method of cumulants produced perverse results for the volatility parameters for diffusion and $J$ in jump-diffusion models of daily stock returns (Press 1967; Beckers, 1981). However, that this application of GMM uses the exact continuous-time moments without approximation and is amenable to non-Gaussian-based distributional assumptions for $J$, such as the Bernoulli mixture of uniform distributions, perhaps recommends the approach as a reference. Nonetheless, the discussion now turns to an alternative that previous literature endorses more enthusiastically.

\section{3. $M L E$}

As noted in the context of GMM, in most cases, there is no closed-form expression for the c.c.f. or, most notably, its Fourier inverse, the conditional probability density function for the short rate. MLE requires some key approximations and can only be applied if $J$ follows a Gaussian form or is a mixture of Gaussians. To begin, consider the corresponding discrete-time expression of the short rate process,

$$
\Delta r=a(b-r) \Delta t+\sigma \Delta W+J \Delta \pi(q),
$$

where the distribution of $J$ is yet to be specified, $\Delta W$ is the discrete-time Weiner increment, and $\Delta \pi(q)$ is the discrete-time Poisson increment. As in Ball and Torous (1983) and Das (1998, 2002), given that the Poisson distribution governs the limit of a Bernoulli distribution, with parameter $q=h \Delta t+O(\Delta t)$, one can approximate the likelihood function for the PoissonGaussian model with a Bernoulli mixture of the normal distributions that characterize the diffusion and jump components of the process. The critical assumption is that during the discrete

\footnotetext{
${ }^{40}$ Results using an alternative $W$, the inverse of the variance-covariance matrix of the sample moments, as well as GMM using conditional as opposed to unconditional moments produce estimates that are even less consistent with intuition than those in Tables 9-11. GMM has not met with considerable success in previous literature - the GMM estimates in Das (1998) were omitted in the published version of the paper (Das, 2002).
} 
time increment, $\Delta t$, either one jump occurs or no jump occurs. Therefore, the assumption is increasingly tenable the higher the frequency of the data.

Under this method and the assumption that $J$ is uni-modal and normally distributed, the approximate transition probability for the short rate is

$$
f_{r}\left(r_{t+\Delta t} \mid r_{t}\right)=(1-q) \frac{\exp \left[\frac{-\left[r_{t+\Delta t}-r_{t}-a\left(b-r_{t}\right) \Delta t\right]^{2}}{2 \sigma^{2} \Delta t}\right]}{\sqrt{2 \pi \sigma^{2} \Delta t}}+q \frac{\exp \left[\frac{-\left[r_{t+\Delta t}-r_{t}-a\left(b-r_{t}\right) \Delta t-\alpha\right]^{2}}{2\left(\sigma^{2} \Delta t+\gamma^{2}\right)}\right]}{\sqrt{2 \pi\left(\sigma^{2} \Delta t+\gamma^{2}\right)}} .
$$

The MLE below extends the method used in Ball and Torous (1983), Das (1998, 2002), and AïtSahalia (2004) to the assumption that jumps follow a Bernoulli mixture of Gaussians. In this case, the approximate transition density function follows

$$
\begin{aligned}
& f_{r}\left(r_{t+\Delta t} \mid r_{t}\right)=(1-q) \frac{\exp \left[\frac{-\left[r_{t+\Delta t}-r_{t}-a\left(b-r_{t}\right) \Delta t\right]^{2}}{2 \sigma^{2} \Delta t}\right]}{\sqrt{2 \pi \sigma^{2} \Delta t}}+q w \frac{\exp \left[\frac{-\left[r_{t+\Delta t}-r_{t}-a\left(b-r_{t}\right) \Delta t-\alpha_{1}\right]^{2}}{2\left(\sigma^{2} \Delta t+\gamma_{1}^{2}\right)}\right]}{\sqrt{2 \pi\left(\sigma^{2} \Delta t+\gamma_{1}^{2}\right)}} \\
& +q(1-w) \frac{\exp \left[\frac{-\left[r_{t+\Delta t}-r_{t}-a\left(b-r_{t}\right) \Delta t-\alpha_{2}\right]^{2}}{2\left(\sigma^{2} \Delta t+\gamma_{1}^{2}\right)}\right]}{\sqrt{2 \pi\left(\sigma^{2} \Delta t+\gamma_{2}^{2}\right)}}
\end{aligned}
$$

That is, the transition density function indicates that there is no jump with probability $1-q$, the first term; there is a jump governed by the first component distribution with probability $q w$, the second term; or there is a jump governed by the second component distribution with probability $q(1-w)$, the third term. (Note that the approximation does not impose that the two modes have the opposite sign.) Also, under the "restricted" assumption that positive and negative jumps are equally likely, that the modes are equal in magnitude but opposite in sign, and the variances around the modes are equal, the density simplifies accordingly.

Given the appropriate density function for either the uni-modal or bi-modal Gaussian assumption, MLE follows

$$
\hat{\Psi}=\max _{\Psi} \sum_{t=1}^{T} \log f\left(r_{t+\Delta t} \mid r_{t}\right),
$$


and the variance-covariance matrix of the estimates follows the Berndt-Hall-Hall-Hausman $(\mathrm{BHHH})$ or outer product of gradients (OPG) method (See Greene, 1997, p. 139; Hamilton, 1994, p. 143).

\subsection{MLE Results}

The MLE covers the same January 4, 1988 - October 12, 2005 period as the GMM estimation as well as the three alternative proxies for the short rate. In addition, again, the approximation of the Poisson with the Bernoulli distribution requires the assumption that no more that one jump occurs during $\Delta t$, and therefore the analyses consider only daily and weekly data. $^{41}$ A reasonable prior is perhaps that higher frequency data are more likely to pick up jumps in the (discrete) short rate process, but the remaining parameters should be insensitive to the frequency.

Turning to the estimates, Table 12 shows the results for the Vasicek (1977) model. ${ }^{42}$ The estimates that use the federal funds rate and one-month bill proxies produce statistically significant estimates with the expected signs and general magnitudes using both daily (Columns 1-2) and weekly data (Columns 4-5). However, the estimates using the three-month bill proxy at either the daily (Column 3) or weekly (Column 6) frequency produce both statistically insignificant estimates of $a$ and $b$ as well as perversely positive and statistically significant estimates of $\sigma$. Turning to jump-diffusion processes, Table 13 includes the results for the model that assume jumps are uni-modal and normally distributed, which is the specification in Das (2002). Each estimate for $a, b, q$, and $\gamma$ is statistically significant and positive across the three proxies for the short rate using both daily and weekly data. Also, an intuitive prior is that the mean jump size is statistically insignificant from zero, and indeed only the weekly estimate for $\alpha$ using the one-month bill (Column 5) is robust, and the parameter suggests that mean jump size is only 5.3 basis points. However, while the estimates of $\sigma$ are positive and statistically significant using daily and weekly data on the federal funds rate and weekly data on one-month bills (Columns 1, 4, and 5), the estimates are perversely negative and statistically significant using daily data on one-month bills and daily and weekly data on three-month bills (Columns 2,

\footnotetext{
${ }^{41}$ Weekly data refer to Wednesday observations, and data cover January 6, 1988 through October 12, 2005.

${ }^{42}$ Of course, the Vasicek (1977) model has a closed-form solution and a closed-form density, and therefore approximations are unnecessary. But for comparison, the estimates follow the approximate technique discussed previously.
} 
3, and 6). Notably, the results using the federal funds rate generally corroborate the findings in Das (2002), who considers a shorter sample from January 1988 through December 1997, but these results broadly suggest that previous findings are somewhat sensitive to either estimation period or short rate proxy.

Table 14 considers the assumption that jumps follow a Bernoulli mixture of Gaussians. As Columns 1-3 indicate, the parameter estimates that use daily data on all three short rate proxies are each statistically significant with the broadly expected magnitude. For example, using the federal funds rate (one-month bill) (three-month bill) the probability of a jump any given day is about 0.137 (0.132) (0.175), and jumps in turn draw roughly evenly between distributions with means of about -66.1 (-67.4) (-87.7) and 25.6 (26.3) (36.8) basis points. However, the corresponding estimates that use weekly data are generally not as robust, although those for $a, b, \sigma$, and $\alpha_{1}$ are statistically significant across the three proxies in Columns 4-6. The estimates of $q, w, \alpha_{2}$, and $\gamma_{2}$ are statistically significant with the expected sign and general magnitude using the federal funds rate, but no other parameter using weekly data is robust.

Finally, Table 15 shows the parameter estimates under the assumption that jumps follow the "restricted” Bernoulli mixture of Gaussians. As Column 1 indicates, daily data using the federal funds proxy produces statistically significant estimates for each parameter, but Columns 2-3 suggest that daily data using one- and three-month bills produce statistically significant and intuitive estimates for $a, b, q, \alpha_{1}$ and $\gamma_{1}$, but the estimates for $\sigma$ are perversely negative and statistically significant. Turning to weekly data, as noted in Column 6, data on the three-month bill produces statistically significant estimates with the generally expected magnitude and sign for each parameter, but the estimates of $\alpha_{1}$ are too close to zero. In addition, while the results in Column 5 using the one-month bill produce significant estimates of $a, b, q$, and $\gamma_{1}$, the estimates of $\sigma$ and $\alpha_{1}$ are similarly incredible, and each parameter estimates expect that for $b$ is statistically insignificant using weekly data on the federal funds rate (Column 4).

In general, the MLE using unconstrained optimization improves on the GMM estimates in that the method produces more statistically significant estimates that broadly accord with intuition, and consistent with Das (2002), the results provide some evidence for jumps in the short rate process. However, there are a few problematic estimates of $\sigma$ that are perversely negative, and the magnitude of the coefficients in some cases is clearly sensitive both to the choice of proxy for the short rate as well as data frequency. 


\subsection{MLE with a Latent Factor}

The applications of GMM and MLE discussed in Tables 9-15, as well those in the literature, such as Das (1998, 2002), estimate the dynamics of the unobservable short rate directly with a proxy. This section outlines an alternative, not found in the literature on ATSMs and jump diffusion processes, in which the short rate remains latent and the parameters are estimated using observable bond yields across the term structure. The procedure is similar to Pearson and Sun (1994), who exploit a known conditional density and a closed-form solution for bond prices, a function of unobservable or latent factors. Using the closed-form expression for bond prices, they express the latent variables in terms of the observable factors and compute the density of the observable factors using the Jacobian of the transformation.

The application below differs from Pearson and Sun (1994) in three respects. First, the analysis uses yields instead of bond prices. Second, the application does not use the exact but rather the approximate densities initially described in Ball and Torous (1983) and employed in the previous section. Third, given that few jump-diffusion models have closed-form solutions, the method uses the (alternative) closed-form approximation of bond prices (yields) that incorporates the two-term Taylor series approximation of the m.g.f. of the appropriate distribution, as outlined in Section 3. That is, in the single-factor case, the density, $f$, for the likelihood function becomes

$$
f_{r}\left(y_{t+\Delta t} \mid y_{t}\right)\left(\frac{\tau}{B(\tau)}\right)
$$

where $y_{t}$ is the yield on a (portfolio of) bond(s). Therefore, $f$ is not evaluated at (the latent factor) $r$, but the short rate can be backed out from the proposed affine solution to the approximate PDDE following 


$$
r=\frac{y \tau+\ln A(\tau)}{B(\tau)}{ }^{43}
$$

Given the transformed density, which again exploits the approximate density and the approximate closed-form solution for bond prices, MLE proceeds as in the previous section.

\subsection{MLE with a Latent Factor: Results}

The estimates under this method use the same January 4, 1988 - October 12, 2005 sample as Tables 9-15. Also, generally following Kim and Wright (2005), $y_{t}$ refers to an equally weighted portfolio of two Treasury bills and six constant maturity zero-coupon yields along an estimated Svensson term structure based on off-the-run Treasury securities. ${ }^{44}$ (The maturity points are three and six months and one, two, four, seven, 10, and 20 years.) Data are sampled at daily and weekly frequencies.

Turning to the results, as Columns 1 and 2 in Table 16 indicate, the magnitude of the estimates using this method for the Vasicek model are largely consistent across daily and weekly data, and each is statistically significant from zero. With respect to jump diffusion processes, as noted in Column 3, the model that assumes jumps are Gaussian and uses daily data only produces statistically significant parameter estimates for $\sigma, q$, and $\gamma$, and as indicated in Column 4 , only $a, b$, and $\sigma$ are robust using weekly data. The results for the model in which jumps follow a Bernoulli mixture of Gaussians are also inconclusive. Given daily data, $a, \sigma, q, \gamma_{1}, \alpha_{2}$, and $\gamma_{2}$ are statistically significant, but the estimate of $b$ is perversely negative and robust, and both $\alpha_{1}$ and $w$ are statistically insignificant (Column 5). Also, as noted in Column 6, the weekly data only produce statistically significant estimates of $\sigma$ and $\gamma_{2}$. Finally, the method using daily data regarding the assumption that jumps follow the "restricted" Bernoulli mixture of Gaussians produces statistically significant estimates for $a, \sigma, q$, and $\gamma_{1}$, as noted in Column 7, but $b$ and $\alpha_{1}$ are not robust. Also, although the estimates of $a$ and $b$ are statistically significant using weekly

\footnotetext{
${ }^{43}$ This follows from the simple relation that $P(r, \tau)=e^{-y \tau}$. Note that the relevant Jacobian (in the single factor case) follows $\frac{d}{d y}[r]=\frac{d}{d y}\left[\frac{y \tau+\ln A(\tau)}{B(\tau)}\right]=\frac{\tau}{B(\tau)}$.

${ }^{44}$ Kim and Wright (2005) do not use the 20-year horizon, and they also account for measurement error.
} 
data, Column 8 indicates that, although perhaps with the expected signs, none of the remaining estimates are robust.

The parameter estimates using GMM and MLE thus far have only addressed the short rate process with respect to the physical measure. Given the presence of $\lambda$ in the closed-form solutions to the approximate PDDE for bond prices, as in (16), the estimates in Tables 9-16, ${ }^{45}$ although perhaps helpful in identifying the presence and the distributional characteristics of jumps, would be of little use in a full application of the model (i.e. for pricing bonds). The riskneutral short rate process follows

$$
d r=[a(b-r)-\lambda \sigma] d t+\sigma d W+J d \pi(h),
$$

and notably, given that the derivation of the bond pricing equation (in the single factor context) requires two bonds, ${ }^{46}$ information from more than one point along the yield curve should inform estimates of $\lambda$. Of course, parameter estimates that rely on a single proxy for the short rate, as in Tables 9-15, do not fulfill this requirement, but the transformed densities based on the portfolio of bonds should permit estimates of $\lambda$.

Toward that end, Table 17 augments the analysis in Table 16 to include estimates of $\lambda$, and the results generally suggest some difficulty in estimating the risk-neutral jump-diffusion model. That is, although $\lambda$ is statistically significant with the expected negative sign in Columns 1,2 , and 4 , the estimate is perversely positive and statistically significant in the daily model that assume jumps follow a Gaussian mixture (Columns 5), and the remaining estimates (Columns 3, 6,7 , and 8) are not robust.

\section{A Two-Factor Jump-Diffusion Model of the Term Structure}

This section extends the analysis in previous sections to consider a second factor, namely a time-varying central tendency, $b$, roughly following Balduzzi et al. (1998), who do not examine jump processes. The dynamics for the (unobservable) state variables in such a two-factor model follow

\footnotetext{
${ }^{45}$ Das (2002) does not provide estimates of the market price of (diffusion) risk.

${ }^{46}$ For example, see Vasicek (1977).
} 


$$
d r=a(b-r) d t+\sigma d W_{r}+J d \pi
$$

and

$$
d b=\kappa(\beta-b) d t+\phi d W_{b}
$$

where $\kappa$ is the reversion rate, $\beta$ is the long-run mean of $b$, and $d W_{r}$ and $d W_{b}$ - the Weiner increments for the short-rate and the central tendency, respectively - are assumed in this simple case to be uncorrelated. Of course, (43) suggests that the short-rate follows a jump diffusion, but its central tendency does not. This formulation might be somewhat problematic in that both state variables ultimately relate to the level of the short rate, yet only $r$ jumps. But, given its relation to a longer-run average, the central tendency is perhaps better characterized by a process that (exclusively) diffuses through all possible points rather than jumps across values. ${ }^{47}$

Again assuming that all time variation is through the state variables, that jump risk is diversifiable, and therefore that the dynamics of the pricing kernel follow

$$
\frac{d \Lambda}{\Lambda}=-r d t-\lambda_{r} d W_{r}-\lambda_{\theta} d W_{b}
$$

where $\lambda_{r}$ and $\lambda_{b}$ denote the market prices of diffusion risk for $r$ and $b$, respectively, the PDDE for bond prices is

$$
0=a(\theta-r) \frac{\partial P}{\partial r}+\frac{1}{2} \sigma^{2} \frac{\partial P}{\partial r^{2}}+\kappa(\beta-\theta) \frac{\partial P}{\partial \theta}+\frac{1}{2} \phi^{2} \frac{\partial P}{\partial \theta^{2}}+h P E_{t}[\exp [-J B(\tau)]-1]-\frac{\partial P}{\partial \tau}-\lambda_{r} \sigma \frac{\partial P}{\partial r}-\lambda_{\theta} \phi \frac{\partial P}{\partial \theta}-P r
$$

Conjecturing an affine solution of the form, $P(r, b, \tau)=A(\tau) \exp [-B(\tau) r-C(\tau) b]$, and noting the correpsonding derivatives, the PDDE reduces to the following system of three ODEs,

$$
\begin{aligned}
& \frac{\partial B}{\partial \tau}+a B=1 \\
& \frac{\partial C}{\partial \tau}+\kappa C=B a
\end{aligned}
$$

and

\footnotetext{
${ }^{47}$ For example, one might expect the short rate to jump in response to economic news or central bank decisions, whereas the central tendency would be more likely to diffuse through all possible points before settling at its new value.
} 


$$
A\left\{\lambda_{r} \sigma B+\frac{1}{2} \sigma^{2} B^{2}+h E_{t}[\exp [-J B(\tau)]-1]-\left(\kappa \beta-\lambda_{b} \phi\right) C+\frac{1}{2} \phi^{2} C^{2}\right\}-\frac{\partial A}{\partial \tau}=0 .
$$

Under the assumption that jumps follow the "restricted" Bernoulli mixture of Gaussians, and given the initial data that $A(0)=1, B(0)=0$, and $C(0)=0$, the closed-form solution to the approximate PDDE follows

$$
\begin{gathered}
B(\tau)=\frac{1-e^{-a \tau}}{a}, \\
C(\tau)=\frac{\kappa-a-\kappa e^{-a \tau}+a e^{-\kappa \tau}}{\kappa(\kappa-a)},
\end{gathered}
$$

and

$$
\begin{aligned}
& A(\tau)=\exp \left\{\left[M_{1}+M_{2}+M_{3}+M_{4}(\kappa-a)+M_{5}\left(\kappa^{2}+a^{2}-2 \kappa a\right)\right] \tau\right. \\
& \quad+\frac{1}{a}\left[M_{1}+2 M_{2}+4 M_{3}+M_{4} \kappa+2 M_{5}\left(\kappa^{2}-a \kappa\right)\right]\left(e^{-a \tau}-1\right) \\
& \quad-\frac{1}{2 a}\left(M_{2}+6 M_{3}+M_{5} \kappa^{2}\right)\left(e^{-2 a \tau}-1\right)+\frac{4 M_{3}}{3 a}\left(e^{-3 a \tau}-1\right)-\frac{M_{3}}{4 a}\left(e^{-4 a \tau}-1\right) \\
& \left.\quad-\frac{a}{\kappa}\left[M_{4}+2 M_{5}(\kappa-a)\right]\left(e^{-\kappa \tau}-1\right)-\frac{a^{2} M_{5}}{2 \kappa}\left(e^{-2 \kappa \tau}-1\right)+\frac{2 M_{5} \kappa a}{\kappa+a}\left(e^{-(\kappa+a) \tau}-1\right)\right\}
\end{aligned}
$$

where $M_{1}=\frac{\lambda_{r} \sigma}{a}, M_{2}=\frac{\sigma^{2}+h\left(\gamma_{1}^{2}+\alpha_{1}^{2}\right)}{2 a^{2}}, M_{3}=\frac{h \gamma_{1}^{4}}{8 a^{4}}, M_{4}=\frac{\lambda_{b} \phi-\kappa \beta}{\kappa(\kappa-a)}$, and $M_{5}=\frac{\phi^{2}}{2 \kappa^{2}(\kappa-a)^{2}} \cdot{ }^{48}$

Estimation of the parameters of the model follows an extension of the MLE method outlined in Section 5.5. Given the (approximate) affine solution in the two-factor case, one can express the latent factors, $r$ and $b$, in terms of the observed yields on two bond portfolios, as in

$$
y_{1}=\frac{-\ln A\left(\tau_{1}\right)+B\left(\tau_{1}\right) r_{t}+C\left(\tau_{1}\right) b_{t}}{\tau_{1}}
$$

\footnotetext{
${ }^{48}$ In the two-factor case, in order for the condition $\lim _{\tau \rightarrow \infty} P(r, b, \tau)=0$ to be met, we must have $M_{1}+M_{2}+M_{3}+M_{4}(\kappa-a)+M_{5}\left(\kappa^{2}+a^{2}-2 \kappa a\right)<0$.
} 
and

$$
y_{2}=\frac{-\ln A\left(\tau_{2}\right)+B\left(\tau_{2}\right) r_{t}+C\left(\tau_{2}\right) b_{t}}{\tau_{2}} .
$$

These equations in turn imply

$$
r_{t}=\frac{\left[y_{1} \tau_{1}+\ln A\left(\tau_{1}\right)\right] C\left(\tau_{2}\right)-\left[y_{2} \tau_{2}+\ln A\left(\tau_{2}\right)\right] C\left(\tau_{1}\right)}{B\left(\tau_{1}\right) C\left(\tau_{2}\right)-B\left(\tau_{2}\right) C\left(\tau_{1}\right)}
$$

and

$$
b_{t}=\frac{\left[y_{2} \tau_{2}+\ln A\left(\tau_{2}\right)\right] B\left(\tau_{1}\right)-\left[y_{1} \tau_{1}+\ln A\left(\tau_{1}\right)\right] B\left(\tau_{2}\right)}{B\left(\tau_{1}\right) C\left(\tau_{2}\right)-B\left(\tau_{2}\right) C\left(\tau_{1}\right)} .
$$

And, the Jacobian of the transformation follows

$$
\left|\begin{array}{ll}
\frac{\partial r_{t}}{\partial y_{1}} & \frac{\partial r_{t}}{\partial y_{2}} \\
\frac{\partial b_{t}}{\partial y_{1}} & \frac{\partial b_{t}}{\partial y_{2}}
\end{array}\right|=\frac{\tau_{1} \tau_{2}}{B\left(\tau_{1}\right) C\left(\tau_{2}\right)-B\left(\tau_{2}\right) C\left(\tau_{1}\right)} .
$$

Therefore, again assuming the Weiner increments of $r$ and $b$ are uncorrelated, the (approximate) density function follows

$$
f_{r}\left(y_{1, t+\Delta t} \mid y_{1, t}\right) f_{b}\left(y_{2, t+\Delta t} \mid y_{2, t}\right) \frac{\tau_{1} \tau_{2}}{B\left(\tau_{1}\right) C\left(\tau_{2}\right)-B\left(\tau_{2}\right) C\left(\tau_{1}\right)},
$$

which permits standard MLE.

Of course, increasing the number of state variables makes parameter estimation more complicated. Table 18 reports estimates for the two-factor model given data and weekly observations from the same sample period in the previous section. Two equally-weighted portfolios are formed for estimation from eight maturity points along the yield curve. The first portfolio includes yields at three and six months and from one to two years, and the second includes yields at four, seven, 10, and 20 years, and similar to Tables 9-17, the results in Columns 1-2 in Table 18 are based on unconstrained optimizations using daily and weekly data, respectively. The daily data produce statistically significant results for each parameter with the expected signs. However, the magnitudes for some of the coefficients are inconsistent with 
intuition, as, for example, the estimate for $q$ seems implausibly low, and those for $\alpha_{1}$ and $\lambda_{b}$ seem excessively large in absolute value. Turning to the weekly data, most of the estimates in Column 2 are statistically insignificant, and among those that are robust, the parameters are too suspiciously close to the starting values in the optimization. ${ }^{49}$

Rather than unconstrained optimizations of the 10 parameters simultaneously, Columns 3-4 present the results from three-stage estimations in which the Vasicek parameters, $a, \sigma$, and $\lambda$; the jump parameters, $q, \alpha_{1}$ and $\gamma_{1}$; and the time-varying central tendency parameters, $\kappa, \beta$, $\phi$, and $\lambda_{b}$ are separately estimated in sequential order using daily and weekly data, respectively. In addition, the optimizations are constrained such that the market prices of risk are non-positive and the remaining parameters are non-negative. Although most of the parameters are robust with the expected sign and general magnitudes, neither estimate for $a$ or $\lambda_{r}$ is statistically significant using daily or weekly data. Moreover, $q$ and $\alpha_{1}$ are not robust using weekly data, and $\lambda_{b}$ is not significant from zero using daily data.

\section{Conclusions}

Incorporating the possibility of non-local changes in the short rate (or other latent factors) considerably complicates both the derivation of solutions for bond prices as well as the estimation of parameters in ATSMs. Regarding the issue of solving the bond pricing equation under jump-diffusion, the preceding analysis expands upon existing linearization techniques that simplify the PDDE in two general ways. First, this paper proposes an alternative approximate PDDE that in some cases seems to provide considerable gains in accuracy. Second, the analysis considers a richer set of presumed distributions for jumps. While previous literature on linearization techniques (Baz and Das, 1996; Ahn and Thompson, 1988) exclusively considers the assumption that jump sizes are normally distributed, this paper considers alternatives, including an exponential/Bernoulli density from Das and Foresi (1996) and the bi-modal Gaussian mixture described previously. The former assumption is particularly useful in that the PDDE has a closed-form solution that enables comparison of the relative accuracy between numerical estimates and the approximate closed-form solutions. Although the choice of parameters is ultimately an arbitrary exercise, the results suggest that the alternative closed-form

\footnotetext{
${ }^{49}$ Specifically, the starting values for the 10 parameters in the two-factor model optimizations are $a=1.525, \sigma=$ $0.05, \lambda=-0.255, q=0.25, \alpha_{1}=0.005, \gamma_{1}=0.0005, \kappa=1.2625, \beta=0.045, \phi=0.005$, and $\lambda_{b}=-0.255$.
} 
approximation (rather than the standard linearization technique) under some specifications produces more accurate estimates of analytical solutions than numerical methods. This finding is generally insensitive to whether the contribution of diffusion or jumps to the overall variance of the short rate process predominates. With respect to other alternative assumptions, the Gaussian mixture follows the intuition that the jump size is bi-modally distributed and that the modes are sufficiently distant from zero. This paper derives two closed-form approximate PDDEs under this distributional assumption, and the parameterizations suggest that the standard linearization technique provides very similar estimates vis-à-vis a numerical solution compared to the approximation that incorporates the m.g.f. of the bi-modal Gaussian mixture.

With respect to parameter estimation of ATSMs that incorporate jumps, the preceding analyses employ GMM and MLE, and applications of the MLE either use direct proxies for the short rate or exploit closed-form solutions to the approximate bond pricing PDDE to express the latent factor(s), the short rate (and its central tendency), in terms of observable variable(s), portfolios of yields. In short, the results from unconstrained optimizations using these methods for the one-factor model are broadly consistent with the presence of jumps, but given sensitivity analyses, the ranges of the remaining parameter estimates seem notably wide. In addition, to extend the analysis to a multi-factor context, this paper outlines a closed-form solution to the approximate PDDE implied by a two-factor model (under which jumps follow a "restricted" bimodal Gaussian mixture).

Beyond the challenges that parameter estimation poses, additional relaxations of the model seem intuitive. For example, one potential possibility is that $h$ is time-varying. Anecdotal evidence suggests that most jumps do not occur at truly random times because monetary policy announcements and economic news releases are largely scheduled, and the jump intensity would seem to be very different during the (instantaneous) interval during which markets process any new information on monetary policy or the economy, compared to "quiet times" during which no announcement or release is on the calendar. ${ }^{50}$ In addition, given the presumed mean-reversion in the short-rate process, jump intensity might be a (deterministic or otherwise) function of $r$ and $b$.

\footnotetext{
${ }^{50}$ For example, Piazzesi (2005) models jump intensity differently on days when the Federal Open Market Committee (FOMC) meets. Also, the results in Johannes (2004) suggest that jumps occur only when the announcements contain significant unexpected components.
} 
Generally speaking, $h$ might increase as the distance between $b$ and $r$ increases. ${ }^{51}$ Moreover, $w$ might be a function of $b$ and $r$, as one might expect positive (negative) jumps to be more likely if the short rate is substantially less than (greater than) its longer-run central tendency. In short, any of these considerations would richen such jump-diffusion models but would of course additionally complicate parameter estimation and their practical application.

\footnotetext{
${ }^{51}$ Irrespective of the longer-run central tendency, $b$, Johannes (2004) finds that jumps are more likely at higher interest rate levels, and he models intensity as a function of the level of the short rate.
} 


\section{References}

Aït-Sahalia, Yacine, 2004, “Disentangling Diffusion from Jumps,” Journal of Financial Economics, vol. 74, pp. 487-528.

Ahn, C.M. and H.E. Thompson, 1988, “Jump-Diffusion Processes and the Term Structure of Interest Rates,” Journal of Finance, vol. 43, no. 1, pp. 155-74.

Balduzzi, P., Sanjiv Ranjan Das, and Silverio Foresi, 1998, “The Central Tendency: A Second Factor in Bond Yields,” The Review of Economics and Statistics, vol. 80, pp. 62-72.

Ball, Clifford A. and Walter N. Torous, 1983, “A Simplified Jump Process for Common Stock Returns,” Journal of Financial and Quantitative Analysis, vol. 18, no. 1, pp. 53-65.

Bates, David S., 1996, “Jumps and Stochastic Volatility: Exchange Rate Processes Implicit in Deutsche Mark Options,” The Review of Financial Studies, vol. 9, no. 1 (Spring), pp. 69-107.

Baz, Jamil, and Sanjiv Ranjan Das, 1996, “Analytical Approximations of the Term Structure for Jump-Diffusion Processes: A Numerical Analysis,” Journal of Fixed Income, pp. 78-86.

Beckers, Stan, 1981, “A Note on Estimating the Parameters of the Diffusion-Jump Model of Stock Returns,” Journal of Financial and Quantitative Analysis, vol. 16 (March), pp. 127-40.

Chapman, D.A., J.B. Long, and N.D. Pearson, 1999, “Using Proxies for the Short Rate: When are Three Months like an Instant?” Review of Financial Studies, vol. 12, no. 4, pp. 763-806.

Cochrane, John H., 2001, Asset Pricing, Princeton University Press.

Cox, J.C., J.E. Ingersoll, and S.A. Ross, 1985, “A Theory of the Term Structure of Interest Rates,” Econometrica, vol. 53, pp. 385-408.

Das, Sanjiv Ranjan, 2002, “The Surprise Element: Jumps in Interest Rates,” Journal of Econometrics, vol. 106, pp. 27-65.

Das, Sanjiv Ranjan, 1998, "Poisson-Gaussian Processes and the Bond Markets,” NBER Working Paper Series, No. 6631.

Das, Sanjiv Ranjan and Silverio Foresi, 1996, “Exact Solutions for Bond and Options Prices with Systematic Jump Risk,” Review of Derivatives Research, vol. 1, pp. 7-24.

Greene, William H., 1997, Econometric Analysis, Third Edition, Prentice-Hall.

Hamilton, James, 1996, “The Daily Market for Federal Funds,” Journal of Political Economy, vol. 104, pp. 26-56.

Hamilton, James, 1994, Time Series Analysis, Princeton University Press. 
Heston, Steven L., 1993, "A Closed-form Solution for Options with Stochastic Volatility with Applications to Bond and Currency Options," The Review of Financial Studies, vol. 6, no. 2, pp. 327-343.

Johannes, Michael, 2004, "The Statistical and Economic Role of Jumps in Continuous-Time Interest Rate Models,” Journal of Finance, vol. 59, pp. 227-260.

Merton, Robert C., 1976, “Option Pricing when Underlying Returns are Discontinuous,” Journal of Financial Economics, vol. 3, pp. 125-144.

Pearson, Neil D. and Tong-Sheng Sun, 1994, "Exploiting the Conditional Density in Estimating the Term Structure: An Application to the Cox, Ingersoll, and Ross Model," Journal of Finance, vol. 54, pp. 1279-1304.

Piazzesi, Monika, 2005, “Bond Yields and the Federal Reserve,” Journal of Political Economy, vol. 113, no. 2, pp. 311-344.

Press, S. James, “A Compound Events Model for Security Prices,” Journal of Business, vol. 40, pp. 317-335.

Vasicek, O., 1977, “An Equilibrium Characterization of the Term Structure of Interest Rates,” Journal of Financial Economics, vol. 5, pp. 177-188. 
Table 1: Jump Size Distribution Assumption: Gaussian

Parameter Assumptions: $a=.1, b=.05, \sigma=.08, \lambda=-.5, \lambda=0, \alpha=0, \gamma=.01, h=10, r=.05$

(1)

(2)

(3)

(4)

(5)

(6)
Numerical

Solution

(Prices)

0.934069236

0.846701167

0.750872691

0.655623186

0.566448506

0.486148003

0.415696372

0.354954352

0.303179960

0.259363390

0.222429111

0.191347643

0.165189644

0.143145416

0.124525321

0.108750798

0.095340799

0.083897829

0.074094599

0.065661928

0.058378382

0.052061706

0.046561605

0.041753854

0.037535481

0.033820859

0.030538500

0.027628730

0.025041131

$\underline{0.022733352}$
Numerical

Solution

(Yields)

0.068204715

0.083203730

0.095506387

0.105542267

0.113673820

0.120207028

0.125400023

0.129470761

0.132603192

0.134952515

0.136649712

0.137805282

0.138512393

0.138849591

0.138883080

0.138668517

0.138252791

0.137675308

0.136969086

0.136161800

0.135276649

0.134332982

0.133346913

0.132331813

0.131298745

0.130256829

0.129213597

0.128174968

0.127146054

$\underline{0.126130740}$
Closed-form

$$
\text { Approx. }
$$

(Prices)

0.934069276

0.846701112

0.750872379

0.655622294

0.566446454

0.486144327

0.415690473

0.354945834

0.303168628

0.259349119

0.222411912

0.191327647

0.165167028

0.143120415

0.124498261

0.108721913

0.095310379

0.083866195

0.074062006

0.065628583

0.058344513

0.052027527

0.046527310

0.041719610

0.037501430

0.033787125

0.030505236

0.027595934

0.025008937

$\underline{0.022701832}$
Closed-form

Approx.

(Yields)

0.068204672

0.083203762

0.095506525

0.105542607

0.113674545

0.120208288

0.125402050

0.129473760

0.132607344

0.134958017

0.136656742

0.137813991

0.138522925

0.138862067

0.138897569

0.138685120

0.138271563

0.137696259

0.136992243

0.136187198

0.135304284

0.134362834

0.133378949

0.132366000

0.131335048

0.130295211

0.129253960

0.128217387

0.127190415

$\underline{0.126176989}$
(7)

Alternativ

Closed-form

|(3)-(5)|

(bps)

0.000428752

0.000320405

0.001386980

0.003401387

0.007245733

0.012601852

0.020272123

0.029994767

0.041529478

0.055023606

0.070300146

0.087088353

0.105320401

0.124763304

0.144885179

0.166028239

0.187717994

0.209514144

0.231569169

0.253979834

0.276349228

0.298514566

0.320357649

0.341866285

0.363029601

0.383819925

0.403637133

0.424186996

0.443604632

0.462489070

$\underline{0.182374231}$

0.934069278

0.846701161

0.750872660

0.655623178

0.566448454

0.486148004

0.415696340

0.354954291

0.303179931

0.259363378

0.222429107

0.191347657

0.165189654

0.143145409

0.124525345

0.108750799

0.095340779

0.083897832

0.074094615

0.065661921

0.058378355

0.052061670

0.046561570

0.041753825

0.037535456

0.033820833

0.030538518

0.027628693

0.025041091

$\underline{0.022733311}$
(8)

(9)

(10)

Alternative

Closed-form

Approx. Approx.

(Prices) (Yields)
0.068204670

0.083203734

0.095506401

0.105542270

0.113673839

0.120207028

0.125400034

0.129470782

0.134952520

0.136649713

0.137805276

0.138512388

0.138849594

0.138883067

0.138668516

0.138252803

0.137675306

0.136969075

0.136161806

0.135276671

0.134333014

0.133346945

0.132331842

0.131298772

0.130256857

0.129213574

0.128175016

0.127146109

$\underline{0.126130799}$
0.132603202

|(3)-(8) |

(bps)

0.000449948

0.000031980

0.000141281

0.000032289

0.000185630

0.000001491

0.000111923

0.000214002

0.000105782

0.000045569

0.000016342

0.000061489

0.000048040

0.000036544

0.000130162

0.000005928

0.000121299

0.000018157

0.000118332

0.000053572

0.000219562

0.000319440

0.000322330

0.000289330

0.000270143

0.000286275

0.000223327

0.000477295

0.000545707 
Table 2: Jump Size Distribution Assumption: Gaussian

Parameter Assumptions: $a=.1, b=.05, \sigma=.02, \lambda=-.5, \lambda_{J}=0, \alpha=0, \gamma=.01, h=16, r=.05$

(1)

(2)

(3)

(4)

(5)

\begin{tabular}{|c|c|c|c|c|}
\hline Maturity & $\begin{array}{l}\text { Numerical } \\
\text { Solution } \\
\text { (Prices) }\end{array}$ & $\begin{array}{l}\text { Numerical } \\
\text { Solution } \\
\text { (Yields) }\end{array}$ & $\begin{array}{l}\text { Closed-form } \\
\text { Approx. } \\
\text { (Prices) }\end{array}$ & $\begin{array}{l}\text { Closed-form } \\
\text { Approx. } \\
\text { (Yields) }\end{array}$ \\
\hline 1 & 0.946931953 & 0.054528044 & 0.946932034 & 0.054527959 \\
\hline 2 & 0.890093038 & 0.058214642 & 0.890093051 & 0.058214635 \\
\hline 3 & 0.832279386 & 0.061195698 & 0.832278967 & 0.061195866 \\
\hline 4 & 0.775426276 & 0.063585592 & 0.775424672 & 0.063586109 \\
\hline 5 & 0.720792857 & 0.065480696 & 0.720788836 & 0.065481812 \\
\hline 6 & 0.669132375 & 0.066962228 & 0.669124278 & 0.066964245 \\
\hline 7 & 0.620834572 & 0.068098660 & 0.620820569 & 0.068101883 \\
\hline 8 & 0.576038273 & 0.068947647 & 0.576016315 & 0.068952412 \\
\hline 9 & 0.534715729 & 0.069557780 & 0.534683774 & 0.069564420 \\
\hline 10 & 0.496734188 & 0.069970023 & 0.496690491 & 0.069978820 \\
\hline 11 & 0.461900080 & 0.070218790 & 0.461842897 & 0.070230045 \\
\hline 12 & 0.429988271 & 0.070333112 & 0.429916290 & 0.070347064 \\
\hline 13 & 0.400762747 & 0.070337360 & 0.400674863 & 0.070354230 \\
\hline 14 & 0.373989263 & 0.070252014 & 0.373884607 & 0.070272005 \\
\hline 15 & 0.349442942 & 0.070094332 & 0.349321222 & 0.070117558 \\
\hline 16 & 0.326913665 & 0.069878698 & 0.326774619 & 0.069905286 \\
\hline 17 & 0.306207471 & 0.069617200 & 0.306051126 & 0.069647242 \\
\hline 18 & 0.287147585 & 0.069319942 & 0.286974197 & 0.069353499 \\
\hline 19 & 0.269574156 & 0.068995356 & 0.269384173 & 0.069032461 \\
\hline 20 & 0.253343458 & 0.068650459 & 0.253137481 & 0.068691127 \\
\hline 21 & 0.238326758 & 0.068291077 & 0.238105516 & 0.068335303 \\
\hline 22 & 0.224409052 & 0.067922035 & 0.224173379 & 0.067969796 \\
\hline 23 & 0.211487778 & 0.067547307 & 0.211238580 & 0.067598568 \\
\hline 24 & 0.199471520 & 0.067170159 & 0.199209764 & 0.067224872 \\
\hline 25 & 0.188278824 & 0.066793252 & 0.188005516 & 0.066851359 \\
\hline 26 & 0.177837086 & 0.066418746 & 0.177553252 & 0.066480181 \\
\hline 27 & 0.168081534 & 0.066048374 & 0.167788205 & 0.066113066 \\
\hline 28 & 0.158954318 & 0.065683515 & 0.158652521 & 0.065751388 \\
\hline 29 & 0.150403702 & 0.065325250 & 0.150094444 & 0.065396226 \\
\hline$\underline{30}$ & $\underline{0.142383314}$ & $\underline{0.064974415}$ & $\underline{0.142067596}$ & $\underline{0.065048410}$ \\
\hline
\end{tabular}

AVERAGE $\underline{0.142383314} \quad \underline{0.064974415}$
(6)

(7)

Alternative

Closed-form

$$
\text { Approx. }
$$

(bps)

0.000855989

0.000072435

0.001676221

0.005170304

0.011157610

0.020169058

0.032222468

0.047649736

0.066403176

0.087971389

0.112552032

0.139515272

0.168704315

0.199911221

0.232258378

0.265886758

0.300419932

0.335562232

0.371053634

0.406682852

0.442260323

0.477610825

0.512611635

0.547128586

0.581065870

0.614349287

0.646919694

0.678730193

0.709760359

0.739948509

0.291876010

(Prices)

0.946932037

0.890093133

0.832279465

0.775426344

0.720792907

0.669132374

0.620834587

0.576038272

0.534715668

0.496734185

0.461900030

0.429988232

0.400762687

0.373989080

0.349442820

0.326913543

0.306207333

0.287147425

0.269573975

0.253343255

0.238326532

0.224408806

0.211487508

0.199471230

0.188278517

0.177836764

0.168081197

0.158953965

0.150403324

$\underline{0.142382921}$
(8)

(9)

(10)

Alternative

Closed-form
Approx.

(Yields)

0.054527955

0.058214589

0.061195666

0.063585570

0.065480683

0.066962228

0.068098657

0.068947647

0.069557793

0.069970024

0.070218800

0.070333120

0.070337372

0.070252049

0.070094356

0.069878721

0.069617226

0.069319973

0.068995391

0.068650499

0.068291122

0.067922085

0.067547363

0.067170219

0.066793317

0.066418816

0.066048448

0.065683595

0.065325337

0.064974507
|(3)-(8)|

(bps)

$0.000533915 \quad 0.136$

$0.000316897 \quad 5.289$

$0.000220253 \quad 23.474$

$0.000138556 \quad 80.528$

$0.000003710 \quad 5437.033$

$0.000033852 \quad 951.873$

$0.000000513 \quad 92878.259$

$0.000125263 \quad 530.109$

$0.000006530 \quad 13472.396$

$0.000097945 \quad 1149.135$

$0.000075526 \quad 1847.239$

$0.000114810 \quad 1469.427$

$0.000348405 \quad 573.790$

$0.000233832 \quad 993.270$

$0.000232091 \quad 1145.616$

$0.000265221 \quad 1132.716$

$0.000310550 \quad 1080.540$

$0.000353631 \quad 1049.268$

$0.000400832 \quad 1014.597$

$0.000452858 \quad 976.599$

$0.000498624 \quad 957.858$

$0.000555123 \quad 923.420$

$0.000605458 \quad 903.661$

$0.000650737 \quad 892.935$

$0.000695447 \quad 883.388$

$0.000742958 \quad 870.736$

$0.000794671 \quad 854.102$

$0.000866079 \quad 819.510$

$\underline{0.000920713} \quad \underline{803.669}$

$0.000382830 \quad \frac{803.669}{4457.384}$ 
Table 3: Jump Size Distribution Assumption: Jump Size (Exponential), Jump Sign (Bernoulli)

Parameter Assumptions: $a=.1, b=.05, \sigma=.08, \lambda=-.5, \lambda_{J}=0, \alpha=200, h=10, r=.05$

(4)

(6)

(7)

(8)

Closed-form Closed-form Numerical Numerical Solution Solution Solution

Solution

(Prices) (Yields) (Prices) $\quad$ (Yields)

$\begin{array}{llll}0.933997016 & 0.068282036 & 0.933997034 & 0.068282016\end{array}$

$\begin{array}{llll}0.846214110 & 0.083491433 & 0.846214122 & 0.083491426\end{array}$

$\begin{array}{lllll}0.653009465 & 0.106540914 & 0.653009530 & 0.106540889\end{array}$

$\begin{array}{lllll}0.653009465 & 0.106540914 & 0.653009530 & 0 & 0.00 \\ 0.562338466 & 0.115130271 & 0.562338517 & 0\end{array}$

$0.480464234-0.122167081-0.480464412$

$\begin{array}{ll}0.122167081 & 0.480464412 \\ 0.12789645 & 0.40845117\end{array}$

$\begin{array}{lll}0.346384594 & 0.132525697 & 0.346384668\end{array}$

0.122167020

0.127896474

$0.293443572-0.136229991 \quad 0.346$

$\begin{array}{llll}0.248683202 & 0.139157547 & 0.248683230\end{array}$

$\begin{array}{llll}0.211026699 & 0.141433692 & 0.211026704\end{array}$

.132525670

0.136229989

$0.179429498 \quad 0.143164410$

0.179429501

$0.152939759-0.144439320$

$\begin{array}{ll}0.130722469 & 0.145334197\end{array}$

0.152939757

0.141433691

0.144439321

$0.112062745 \quad 0.145913089$

0.130722467

0.145334198

0.096358284

0.112062744

0.145913090

$\begin{array}{ll}0.083106945 & 0.146331000\end{array}$

0.083106945

0.146230120

$0.071892897 \quad 0.146254323$

$0.062373163 \quad 0.146032640$

0.071892897

0.146331000

0.146254322

0.062373164

0.146032640

0.047337459

$\begin{array}{ll}0.041398082 & 0.144750942\end{array}$

0.054265434

0.145693391

$\begin{array}{lll}0.036289743 & 0.144183484\end{array}$

$0.031882319 \quad 0.143570986$

$0.028068082 \quad 0.142924890$

$0.024757588 \quad 0.142254740$

$0.021876354 \quad 0.141568479$

$0.019362146 \quad 0.140872691$

\begin{tabular}{ll}
0.017162791 & 0.140172813 \\
\hline
\end{tabular}

$\underline{0.015234401}$

$\underline{0.139473307}$

$0.047337460 \quad 0.145259683$

$\begin{array}{ll}0.041398085 & 0.144750939 \\ 0.036289744 & 0.144183483\end{array}$

0.031882319

0.144183483

0.02806808

0.142924890

0.021876354

0.142254741

0.019362146

0.141568479

0.017162791

0.140172813

$\underline{0.139473307}$

\begin{tabular}{|c|c|c|}
\hline $\begin{array}{c}|(3)-(5)| \\
\text { (bps) }\end{array}$ & $\begin{array}{l}\text { Closed-form } \\
\text { Approx. } \\
\text { (Prices) }\end{array}$ & $\begin{array}{l}\text { Closed-form } \\
\text { Approx. } \\
\text { (Yields) }\end{array}$ \\
\hline 00196531 & 0.933997015 & 0.068282037 \\
\hline 0072620 & 0.846214085 & 0.083491448 \\
\hline 0184047 & 0.749516286 & 0.096109077 \\
\hline 0251077 & 0.653009025 & 0.106541082 \\
\hline 00181843 & 0.562337473 & 0.115130625 \\
\hline 0618196 & 0.480462417 & 0.122167712 \\
\hline 00006128 & 0.408492232 & 0.127897483 \\
\hline 000269145 & 0.346380465 & 0.132527187 \\
\hline 000017346 & 0.293438099 & 0.136232063 \\
\hline 00113423 & 0.248676361 & 0.139160298 \\
\hline 00019364 & 0.211018535 & 0.141437210 \\
\hline 00011277 & 0.179420107 & 0.143168771 \\
\hline 000010463 & 0.152929275 & 0.144444594 \\
\hline 000012891 & 0.130711044 & 0.145340440 \\
\hline 000008012 & 0.112050543 & 0.145920349 \\
\hline 000002705 & 0.096345470 & 0.146238431 \\
\hline 000001291 & 0.083093677 & 0.146340392 \\
\hline 000003344 & 0.071879322 & 0.146264814 \\
\hline 000003619 & 0.062359417 & 0.146044241 \\
\hline 000015942 & 0.054251635 & 0.145706108 \\
\hline 000006996 & 0.047323715 & 0.145273511 \\
\hline 000028720 & 0.041384484 & 0.144765875 \\
\hline 000007475 & 0.036276368 & 0.144199512 \\
\hline 000001162 & 0.031869232 & 0.143588094 \\
\hline 000001563 & 0.028055335 & 0.142943059 \\
\hline 000009683 & 0.024745226 & 0.142273950 \\
\hline 00004573 & 0.021864409 & 0.141588708 \\
\hline 00000757 & 0.019350643 & 0.140893915 \\
\hline 00000412 & 0.017151748 & 0.140195007 \\
\hline 000000992 & 0.015223829 & $\underline{0.139496445}$ \\
\hline
\end{tabular}

0.000068720

$\underline{0.015223829} \quad \underline{0.139496445}$

AVERAGE

$\underline{0.231379809}$

0.091259127
Alternative

(11)

Alternative

$\begin{array}{ccc} & & \\ \text { (3)-(8)| } & \text { Approx. } & \text { Approx. }\end{array}$

(bps) (Prices) (Yields)

(bps)

0.068282036

$\begin{array}{ll}0.933997016 & 0.068282036 \\ 0.846214110 & 0.083491433 \\ 0.749516426 & 0.096109015\end{array}$

0.000000000

0.000000008

0.000000076

$\begin{array}{llll}0.001684882 & 0.653009465 & 0.106540914 & 0.000000333 \\ 0.003531050 & 0.562338466 & 0.115130272 & 0.000000998\end{array}$

$\begin{array}{llll}0.006304027 & 0.480464233 & 0.122167082 & 0.000002355\end{array}$

$\begin{array}{lllll}0.010084814 & 0.408495114 & 0.127896475 & 0.000004714\end{array}$

$\begin{array}{lllll}0.014898757 & 0.346384591 & 0.132525698 & 0.000008374\end{array}$

$\begin{array}{llll}0.020725446 & 0.293443569 & 0.136229992 & 0.000013598\end{array}$

$\begin{array}{lllll}0.027509605 & 0.248683197 & 0.139157550 & 0.000020586\end{array}$

$\begin{array}{lllll}0.035171372 & 0.211026692 & 0.141433695 & 0.000029469\end{array}$

$\begin{array}{llll}0.043615230 & 0.179429489 & 0.143164414 & 0.000040310\end{array}$

$\begin{array}{llll}.052737323 & 0.152939749 & 0.144439326 & 0.000053102\end{array}$

$\begin{array}{llll}.072591926 & 0.112062731 & 0.145913098 & 0.000084256\end{array}$

$\begin{array}{llll}0.083119451 & 0.096358268 & 0.146230130 & 0.000102368\end{array}$

$\begin{array}{lllll}0.093920305 & 0.083106928 & 0.146331013 & 0.000121958\end{array}$

$\begin{array}{lllll}0.0 .07468996 & 0.071892879 & 0.146254337 & 0.000142845\end{array}$

$\begin{array}{llll}0.116008594 & 0.062373144 & 0.146032657 & 0.000164843\end{array}$

$\begin{array}{lllll}0.127150896 & 0.054265412 & 0.145693411 & 0.000187765\end{array}$

$\begin{array}{llll}0.047337438 & 0.145259704-0.000211429\end{array}$

$\begin{array}{lllll}0.149333225 & 0.041398061 & 0.144750965 & 0.000235662\end{array}$

$\begin{array}{llll}0.160277965 & 0.036289722 & 0.144183510 & 0.000260305\end{array}$

$\begin{array}{llll}0.171073686 & 0.031882298 & 0.143571015 & 0.000285208\end{array}$

$\begin{array}{lll}0.028068060 & 0.142924921 & 0.000310240\end{array}$

$\begin{array}{lllll} & 1.02102104 & 0.024757567 & 0.142254773 & 0.000335279\end{array}$

$\begin{array}{llll}0.0 .14254753 & 0.00335279 \\ 0.202290452 & 0.021876333 & 0.141568515 & 0.000360222\end{array}$

$\begin{array}{lll}0.019362125 & 0.140872730 & 0.000384978\end{array}$

\begin{tabular}{ll}
0.017162770 & 0.140172854 \\
\hline
\end{tabular}

0.000384978

$\underline{0.000433622}$

0.000142405 
Table 4: Jump Size Distribution Assumption: Jump Size (Exponential), Jump Sign (Bernoulli)

Parameter Assumptions: $a=.1, b=.05, \sigma=.02, \lambda=-.5, \lambda_{J}=0, \alpha=200, h=16, r=.05$

(4)

(5)

(6)

(7)

(8)

rnative

Alternative

Closed-form

Solution

(Prices)

Solution

Numerical
Solution

Numerical

(Prices) (Yields)

0.946814828

054651741

Yields)

0.054651751

$\begin{array}{lllll}0.770486148 & 0.065183400 & 0.770486149 & 0.065183400\end{array}$

$\begin{array}{lll}0.712443352 & 0.067810975 & 0.712443389\end{array}$

$\begin{array}{llll}0.656659352 & 0.070098314 & 0.656659352\end{array}$

$\begin{array}{lll}0.656659352 & 0.070098314 & 0.656659352 \\ 0.603716397 & 0.072092962 & 0.603716400\end{array}$

$\begin{array}{lll}0.553948203 & 0.073835512 & 0.553948261\end{array}$

$\begin{array}{lll}0.507506434 & 0.075360654 & 0.507506495\end{array}$

$\begin{array}{lll}0.464413165 & 0.076698068 & 0.464413203\end{array}$

$\begin{array}{lll}0.424601232 & 0.077873166 & 0.424601242\end{array}$

$0.387944573-0.078907734-0.387944568$

$\begin{array}{lll}0.354280601 & 0.079820463 & 0.354280598 \\ 0.323426375 & 0.080627413 & 0.323426380\end{array}$

$\begin{array}{llll}0.295190057 & 0.081342391 & 0.295190088\end{array}$

$\begin{array}{lll}0.269378838 & 0.081977286 & 0.269378839\end{array}$

$\begin{array}{lll}0.245804245 & 0.082542342 & 0.245804241\end{array}$

$\begin{array}{lll}0.224285564 & 0.083046400 & 0.224285571\end{array}$

$\begin{array}{llll}0.204651890 & 0.083497097 & 0.204651902\end{array}$

$\begin{array}{lll}0.186743222 & 0.083901038 & 0.186743229 \\ 0.170410886 & 0.084263942 & 0.170410886\end{array}$

$\begin{array}{lll}0.155517515 & 0.084590769 & 0.155517511\end{array}$

$\begin{array}{lll}0.141936719 & 0.084885824 & 0.141936717\end{array}$

$\begin{array}{llll}0.129552586 & 0.085152850 & 0.129552586\end{array}$

$0.118259062 \quad 0.085395105$

$\begin{array}{ll}0.107959286 & 0.085615427\end{array}$

0.118259067

0.107959282

$\begin{array}{lll}0.098564909 & 0.085816295 & 0.098564910\end{array}$

$\begin{array}{lll}0.089995419 & 0.085999875 & 0.089995417\end{array}$

$\begin{array}{lll}0.082177493 & 0.086168063 & 0.082177490\end{array}$

$\begin{array}{lll}\underline{0.075044381} & \underline{0.086322520} & \underline{0.075044378}\end{array}$

0.070098314

.072092961

0.075360641

0.076698060

0.077873164

0.079820464

0.080627412

0.081342384

0.082542343

0.083046398

0.083497094

0.083901036

0.084590770

0.084885825

|(3)-(5)|

Closed-for

(bps)

(Prices)

Approx.

$\begin{array}{ll}0.946814826 & 0.054651742\end{array}$

|(3)-(8)|

$\begin{array}{lll}\text { (3)-(8) } & \text { Approx. } & \text { Approx. } \\ \text { (bps) } & \text { (Prices) } & \text { (Yields) }\end{array}$

Closed-form

Yields)

$\begin{array}{lll}0.000016958 & 0.946814828 & 0.054651741\end{array}$

$\begin{array}{lllllll}0.000009841 & 0.889274014 & 0.058674932 & 0.000230754 & 0.889274056 & 0.058674909\end{array}$

$\begin{array}{llll}0.000028293 & 0.829875287 & 0.062159949 & 0\end{array}$

$0.000996681 \quad 0.829875535$

0.062159849

$\begin{array}{ll}0.770485318 & 0.065183670\end{array}$

$0.002695812 \quad 0.770486148$

0.065183400

$0.000101969 \quad 0.712441340$

0.067811540

$0.000002001 \quad 0.656655378$

0.070099323

0.712443352

$\begin{array}{ll}0.000009084 & 0.603709578 \\ 0.000130368 & 0.553937640\end{array}$

0.072094575

0.070098314

0.072092963

0.075363971

$0.000133923 \quad 0.507491288$

$0.000082633 \quad 0.464392724$

$\begin{array}{ll}0.016135702 & 0.603716393 \\ 0.023838011 & 0.553948198\end{array}$

$\begin{array}{lll}0.033160714 & 0.507506424 & 0.07\end{array}$

$\begin{array}{ll}0.000023328 & 0.424574949 \\ 0.000011176 & 0.387912088\end{array}$

0.07787879

$0.044015368 \quad 0.464413150$

0.075360657

0.076698071

0.077873171

0.078914712

$0.000006014 \quad 0.35$

0.354241741

0.079828901

$\begin{array}{ll}0.056274195 & 0.424601210\end{array}$

$\begin{array}{ll}0.084379716 & 0.354280562\end{array}$

0.000068405

0.295138634

0.080637402

0.078907740

0.079820472

$\begin{array}{llll}0.000002867 & 0.269321524 & 0.08194006\end{array}$

0.323426326
0

0.080627424

0.081342405

$\begin{array}{ll}0.245741459 & 0.082557369\end{array}$

$0.000018249 \quad 0.224217809$

0.083063186

0.245804163

$\begin{array}{ll}0.000030613 & 0.204579729\end{array}$

0.083515658

0.082542361

0.083046423

0.186667254

0.00000008

0.170331731

0.083921382

$\begin{array}{ll}0.167854393 & 0.224285471 \\ 0.185613750 & 0.204651787\end{array}$

0.083497123

0.083901068

0.000012713

0.155435788

0.084286066

0.18674310

0.00000833

0.141853027

0.084614662

0.17041076

0.084263976

0.084590807

0.129467508

0.085395103

0.118173147

0.085180222

$0.273717898 \quad 0.129552444$

.084885866

0.085615429

0.000013357

0.107873045

0.085646164

0.129552444

0.085152896

0.09847881

0.085848662

0.107959135

0.085395155

0.085615481

0.085999876

0.000006756

0.0861680640 .000010506

0.082092910

0.086033834

$\begin{array}{ll}0.323664724 & 0.098564755\end{array}$

0.085816353

0.089995264

0.35510228

0.082177337

0.085999937

$\underline{0.000012782}$

$\underline{0.086359540}$

$\underline{0.370207694}$

0.086168128

0.146014603

AVERAGE

0.000029764

|(3)-(11)|

0.000000001

(6)/(12)

71159.182

711.046

233.181

3.725

63.823

0.531

1.205

9.730

6.155

2.509

0.495

0.173

0.071

0.095

0.507

0.018

0.049

0.080

0.116

0.067

0.000

0.034

0.020

0.001

0.039

0.025

0.012

0.011

0.016

$\underline{0.018}$

2406.431
(9)/(12)

11922.551

16672.487

8214.244

5059.827

3536.185

2676.603

1779.063

1524.112

1336.312

1193.482

1082.005

993.127

920.997

861.568

811.967

770.104

734.424

703.752

677.182

654.009

633.675

615.732

599.820

585.644

572.961

561.571

551.304

542.020

$\frac{533.597}{315.326}$

2315.326 
Table 5: Jump Size Distribution Assumption: Gaussian Mixture

Parameter Assumptions: $a=.1, b=.05, \sigma=.08, \lambda=-.5, \lambda_{J}=0, \alpha_{1}=.006, \gamma_{1}=.0015, \alpha_{2}=-.004, \gamma_{2}=.001, w=.4, h=10, r=.05$

(1)

$$
\text { (2) }
$$

(6)

(7)

(8)

(9)

Alternative

Alternative

Closed-form Closed-form

Solution

(Prices)

0.933961543

0.845975278

0.748851687

0.651729871

0.560330319

0.477695296

0.405000382

0.342245780

0.288768415

0.243589169

0.205629457

0.173835767

Numerical

Solution

Approx.

Approx.

0.068320016

0.083632571

0.096404777

0.107031278

0.115845763

0.123130368

0.129123896

0.134028268

0.138014472

0.141227221

0.143789044

0.147243537

0.145803691

0.125003693

0.147359027

0.106387431

0.148529429

0.090779370

0.149377856

0.077665895

149957702

0.066621250

0.150314062

0.057293948

0.150485094

0.049394746

0.042685685

0.150395561

0.036970986

0.150185317

0.032089393

0.149891902

0.027907863

0.149531728

0.024316387

0.149118617

0.021223771

0.148664192

0.148178209

018554207

0.147668841

0.016244451

0.147142996

0.014241758

0.146606100

30

$\underline{0.012501667}$

$\underline{0.146063110}$

$$
\text { (Prices) }
$$

0.933961609

0.845975544

0.748852695

0.651732328

0.560334967

0.477702571

0.405010730

0.342259201

0.288784779

0.243608181

0.173858877

0.147268069

0.125029221

0.106413518

0.090805733

0.077692230

0.066647263

0.057319483

0.049419635

0.042709792

0.036994204

0.032111646

0.027929101

0.024336583

0.021242915

0.018572304

0.016261554

0.014257810

$\underline{0.012516727}$
0.205650731
Yields)

0.068319946

0.083632414

0.096404328

0.107030335

0.115844104

0.123127829

0.129120245

0.134023367

0.138008175

0.141219416

0.143779639

0.145792614

0.147346212

0.148514843

0.149361511

0.149939554

0.150294119

0.150463405

0.150479720

0.150370373

0.150158432

0.149863365

0.149501587

0.149086920

0.148630984

0.148143532

0.147632734

0.147105414

0.146567257

$\underline{0.146022979}$

$$
\begin{gathered}
|(3)-(5)| \\
\text { (bps) }
\end{gathered}
$$

Approx.

$$
\text { (Prices) }
$$

0.000707134

0.001574248

0.004487965

0.009423686

0.016591326

0.025381651

0.036502031

0.049016585

0.062962581

0.078044571

0.094048970

0.110773602

0.128150375

0.145855144

0.163454443

0.199429595

0.216882633

0.234525264

0.251875428

0.268845427

0.285364882

0.301409056

0.332085291

0.346768283

0.361065013

0.375816420

0.388434913

$\underline{0.401313488}$

0.179641423
0.181477199

0.316975495
0.933961606

0.845975504

0.748852535

0.651731934

0.560334227

0.477701396

0.405009071

0.342257044

0.288782147

0.243605119

0.205647300

0.173855145

0.147264107

0.125025096

0.106409293

0.090801463

0.077687964

0.066643040

0.057315338

0.049415594

0.042705877

0.036990431

0.032108027

0.027925644

0.024333293

0.021239794

0.018569352

0.016258770

0.014255190

$\underline{0.012514268}$ pprox.

(Yields)

0.068319949

0.083632437

0.096404399

0.107030487

0.115844368

0.123128239

0.129120831

0.134024154

0.138009188

0.141220673

0.143781156

0.145794402

0.147348281

0.148517199

0.149364158

0.149942493

0.150297349

0.150466925

0.150483527

0.150374462

0.150162797

0.149868002

0.149506488

0.149092078

0.148636392

0.148149184

0.147638621

0.147111530

0.146573593

$\underline{0.146029528}$$$
\text { (bps) }
$$

0.000673842

0.001337272

0.003774745

0.007912785

0.013948318

0.021282488

0.030647478

0.041140383

0.052835263

0.065474340

0.078880660

0.092886444

0.107455075

0.122290636

0.136984555

0.152087392

0.167123876

0.181680696

0.196459896

0.210990186

0.225192576

0.239003595

0.252403845

0.265394861

0.278000569

0.290252665

0.302192720

0.314662018

0.325072741

$\underline{0.335817191}$

0.150461970

(6)/(9)

1.049

1.177

1.189

1.191

1.189

1.193 
Table 6: Jump Size Distribution Assumption: Gaussian Mixture

Parameter Assumptions: $a=.1, b=.05, \sigma=.02, \lambda=-.5, \lambda_{J}=0, \alpha_{1}=.006, \gamma_{1}=.0015, \alpha_{2}=-.004, \gamma_{2}=.001, \mathrm{w}=.4, h=31, r=.05$

(1)

(2)

(3)

(4)

(5)

(6)

(7)

(8)

Alternative Alternative

Closed-form Closed-form

Numerical Numerical Closed-form Closed-form

Solution Solution Approx. Approx.

(Prices)

0.946813334

0.889263445

0.829843310

0.770417818

0.712324541

0.656477452

0.603461496

0.553613333

0.507087788

0.463909922

0.424014997

0.387279296

0.353541648

0.322620467

0.294324786

0.268462306

0.244844762

0.223291393

0.203631088

0.185703434

0.169359350

0.154460905

0.140881124

0.128503506

0.117221388

0.106937294

0.097562303

0.089015373

0.081222681

$\underline{0.074116961}$
(Prices)

0.054653318

0.058680874

0.062172793

0.065205572

0.067844331

0.070144489

0.072153292

0.073911099

0.075452349

0.076806488

0.077998769

0.079050763

0.079981076

0.080805619

0.081538094

0.082190298

0.082772406

0.083293204

0.083760280

0.084180216

0.084558690

0.084900648

0.085210384

0.085491629

0.085747637

0.085981256

0.086194967

0.086390936

0.086571060

$\underline{0.086737029}$
0.946813434

0.889264293

0.829846785

0.770426852

0.712342796

0.656508715

0.603509244

0.553680605

0.507176829

0.464022085

0.424150960

0.387438779

0.353723987

0.322824362

0.294548507

0.268703815

0.245101833

0.223561712

0.203912300

0.185993264

0.169655544

0.154761348

0.141183846

0.128806679

0.117523356

0.107236591

0.097857631

0.089305584

0.081506773

$\underline{0.074394125}$
(Yields)

0.054653212

0.058680398

0.062171397

0.065202641

0.067839206

0.070136552

0.072141989

0.073895910

0.076782313

0.077969623

0.079016453

0.079941413

0.080760491

0.081487439

0.082134098

0.082710677

0.083225988

0.083687646

0.084102241

0.084475481

0.084812320

0.085117059

0.085393442

0.085644728

0.085873760

0.086083022

0.086274688

0.086450660

0.086612610
0.075432840

\begin{tabular}{c}
$|(3)-(5)|$ \\
$\underline{(\mathrm{bps})}$ \\
0.001062380 \\
0.004767323 \\
0.013955096 \\
0.029314439 \\
0.051254263 \\
0.079369294 \\
0.113028317 \\
0.151883803 \\
0.195084647 \\
0.241748614 \\
0.291460320 \\
0.343097374 \\
0.396629142 \\
0.451283710 \\
0.506551640 \\
0.561999583 \\
0.617286804 \\
0.672155737 \\
0.726335107 \\
0.779749530 \\
0.832085303 \\
0.883280139 \\
0.933246919 \\
0.981868230 \\
1.029093854 \\
1.074961048 \\
1.119445386 \\
1.162477288 \\
1.203997740 \\
1.244190704 \\
\hline 0.556422124 \\
\hline
\end{tabular}

Approx.

(Prices)

0.946813425

0.889264162

0.829846234

0.770425409

0.712339878

0.656503709

0.603501577

0.553669790

0.507162499

0.464004004

0.424129022

0.387412999

0.353694487

0.322791349

0.294512254

0.268664648

0.245060108

0.223517803

0.203866587

0.185946123

0.169607338

0.154712422

0.141134524

0.128757258

0.117474106

0.107187755

0.097809423

0.089258192

0.081460358

$\underline{0.074348824}$
(Yields)

0.054653223

0.058680471

0.062171618

0.067840025

0.070137823

0.072143804

0.073898352

0.075435980

0.076786210

0.077974325

0.079021998

0.079947828

0.080767796

0.081495644

0.082143209

0.082720692

0.083236901

0.083699447

0.084114915

0.084489014

0.084826692

0.08513225

0.085409432

0.085661494

0.085891279

0.086101273

0.086293646

0.086470303

$\underline{0.086632914}$
0.065203109
(9)

$$
\text { |(3)-(8)| }
$$

(bps)

0.000959176

0.004032697

0.011744114

0.024630644

0.043060938

0.066661887

0.094879204

0.127467579

0.163689961

0.202780896

0.244438559

0.287647183

0.332473711

0.378233734

0.424494988

0.470891184

0.517139075

0.563029731

0.608332466

0.653005278

0.696761465

0.739560151

0.781330766

0.821968264

0.861431216

0.899762630

0.936941278

0.972898642

1.007575006

1.041152182

0.465965820
(6)/(9)

1.108

1.182

1.188

1.190

1.190

1.191

1.191

1.192

1.192

1.192

1.192

1.193

1.193

1.193

1.193

1.193

1.194

1.194

1.194

1.194

1.194

1.194

1.194

1.195

1.195

1.195

1.195

1.195

1.195

$\underline{1.195}$ 
Table 7: Alternative Distributional Assumptions for Jumps

Parameter Assumptions: $a=.1, b=.05, \sigma=.08, \lambda=-.5, \lambda_{J}=0, \alpha=0, \gamma=.01, h=10, r=.05$

(1)

(2)

(3)

(4)

(5)

(6)

Restricted

\begin{tabular}{|c|c|c|c|c|c|c|c|}
\hline Maturity & $\begin{array}{l}\text { Gaussian } \\
\text { (Yields) }\end{array}$ & $\begin{array}{c}\text { Exponential } \\
\text { (Yields) }\end{array}$ & $\begin{array}{l}\text { Gaussian } \\
\text { Mixture } \\
\text { (Yields) }\end{array}$ & $\begin{array}{l}\text { Gaussian } \\
\text { Mixture } \\
\text { (Yields) }\end{array}$ & $\begin{array}{c}|(2)-(3)| \\
\text { (bps) }\end{array}$ & $\begin{array}{c}|(2)-(4)| \\
\underline{(b p s)}\end{array}$ & $\begin{array}{c}|(2)-(5)| \\
\underline{(b p s)}\end{array}$ \\
\hline 1 & 0.068204670 & 0.068204668 & 0.068204694 & 0.068204671 & 0.000021196 & 0.000242376 & 0.000009273 \\
\hline 2 & 0.083203734 & 0.083203705 & 0.083203912 & 0.083203746 & 0.000288425 & 0.001783383 & 0.000126186 \\
\hline 3 & 0.095506401 & 0.095506276 & 0.095506954 & 0.095506455 & 0.001245699 & 0.005526912 & 0.000544993 \\
\hline 4 & 0.105542270 & 0.105541933 & 0.105543472 & 0.105542417 & 0.003369098 & 0.012016760 & 0.001473981 \\
\hline 5 & 0.113673839 & 0.113673133 & 0.113675990 & 0.113674147 & 0.007060103 & 0.021513576 & 0.003088795 \\
\hline 6 & 0.120207028 & 0.120205768 & 0.120210434 & 0.120207579 & 0.012603343 & 0.034064835 & 0.005513963 \\
\hline 7 & 0.125400034 & 0.125398018 & 0.125404991 & 0.125400916 & 0.020160200 & 0.049564714 & 0.008820088 \\
\hline 8 & 0.129470782 & 0.129467804 & 0.129477562 & 0.129472085 & 0.029780764 & 0.067803170 & 0.013029084 \\
\hline 9 & 0.132603202 & 0.132599060 & 0.132612053 & 0.132605014 & 0.041423695 & 0.088504781 & 0.018122867 \\
\hline 10 & 0.134952520 & 0.134947022 & 0.134963655 & 0.134954925 & 0.054978037 & 0.111358544 & 0.024052891 \\
\hline 11 & 0.136649713 & 0.136642685 & 0.136663317 & 0.136652788 & 0.070283804 & 0.136040041 & 0.030749164 \\
\hline 12 & 0.137805276 & 0.137796561 & 0.137821499 & 0.137809089 & 0.087149841 & 0.162227367 & 0.038128056 \\
\hline 13 & 0.138512388 & 0.138501851 & 0.138531349 & 0.138516998 & 0.105368441 & 0.189612094 & 0.046098693 \\
\hline 14 & 0.138849594 & 0.138837122 & 0.138871385 & 0.138855051 & 0.124726760 & 0.217906397 & 0.054567957 \\
\hline 15 & 0.138883067 & 0.138868566 & 0.138907752 & 0.138889412 & 0.145015341 & 0.246847258 & 0.063444212 \\
\hline 16 & 0.138668516 & 0.138651913 & 0.138696136 & 0.138675780 & 0.166034167 & 0.276198511 & 0.072639948 \\
\hline 17 & 0.138252803 & 0.138234043 & 0.138283378 & 0.138261010 & 0.187596695 & 0.305751335 & 0.082073554 \\
\hline 18 & 0.137675306 & 0.137654353 & 0.137708838 & 0.137684473 & 0.209532301 & 0.335323659 & 0.091670382 \\
\hline 19 & 0.136969075 & 0.136945906 & 0.137005550 & 0.136979211 & 0.231687502 & 0.364758849 & 0.101363282 \\
\hline 20 & 0.136161806 & 0.136136413 & 0.136201198 & 0.136172915 & 0.253926263 & 0.393923942 & 0.111092740 \\
\hline 21 & 0.135276671 & 0.135249058 & 0.135318942 & 0.135288752 & 0.276129666 & 0.422707636 & 0.120806729 \\
\hline 22 & 0.134333014 & 0.134303195 & 0.134378116 & 0.134346060 & 0.298195126 & 0.451018176 & 0.130460367 \\
\hline 23 & 0.133346945 & 0.133314942 & 0.133394824 & 0.133360947 & 0.320035320 & 0.478781241 & 0.140015452 \\
\hline 24 & 0.132331842 & 0.132297684 & 0.132382436 & 0.132346786 & 0.341576955 & 0.505937905 & 0.149439918 \\
\hline 25 & 0.131298772 & 0.131262496 & 0.131352017 & 0.131314643 & 0.362759458 & 0.532442704 & 0.158707263 \\
\hline 26 & 0.130256857 & 0.130218504 & 0.130312684 & 0.130273637 & 0.383533650 & 0.558261855 & 0.167795972 \\
\hline 27 & 0.129213574 & 0.129173188 & 0.129271912 & 0.129231243 & 0.403860460 & 0.583371624 & 0.176688951 \\
\hline 28 & 0.128175016 & 0.128132645 & 0.128235792 & 0.128193553 & 0.423709701 & 0.607756857 & 0.185372994 \\
\hline 29 & 0.127146109 & 0.127101803 & 0.127209250 & 0.127165493 & 0.443058925 & 0.631409659 & 0.193838280 \\
\hline$\underline{30}$ & $\underline{0.126130799}$ & $\underline{0.126084610}$ & $\underline{0.126196232}$ & $\underline{0.126151007}$ & $\underline{0.461892372}$ & $\underline{0.654328235}$ & $\underline{0.202077913}$ \\
\hline & & & & & 0.182233444 & 0.281566146 & 0.079727132 \\
\hline
\end{tabular}

(8)

-(5)

273 . 
Table 8: Alternative Distributional Assumptions for Jumps

Parameter Assumptions: $a=.1, b=.05, \sigma=.08, \lambda=-.5, \lambda_{J}=0, \alpha=0, \gamma=.005, h=50, r=.05$
(1)

(2)
(3)
(4)

(5)

(6)

Restricted

Gaussian

Mixture

(Yields)

0.068165989

0.083059920

0.095205250

0.105043369

0.112946505

0.119228576

0.124154334

0.127947046

0.130794985

0.132856877

0.134266508

0.135136601

0.134231286

0.133405434

0.132459004

0.131419809

0.130311041

0.129151994

0.127958667

0.126744278

0.125519707

0.124293856

0.123073966

0.121865882

0.120674273

$\underline{0.119502826}$

0.135615085

0.135377115

0.134898087

0.134225423

0.133398886

0.132451764

0.131411873

0.130302412

0.129142676

0.127948665

0.126733604

0.125508371

0.124281870

0.123061345

0.121852641

0.120660428

$\underline{0.119488392}$
0.135562090

0.135622880

0.135386179

0.134908464

0.135570535

0.135632496

0.134250331

0.133426347

0.132481778

0.134237148

0.133411982

0.132466244

$0.131444429 \quad 0.131427744$

0.130337485

0.129180233

0.127988667

0.126776003

0.125553116

0.124328906

0.123110614

0.121904081

0.120713979

$\underline{0.119543991}$
0.130319670

0.129161313

0.127968668

0.126754953

0.125531043

0.124305841

0.123086587

0.121879123

$\underline{0.119517260}$
0.120688119

AVERAGE

$$
\text { |(2)-(3)| }
$$$$
\text { (bps) }
$$

0.000006624

0.000090133

0.000389281

0.001052843

0.002206282

0.003938545

0.006300063

0.009306489

0.012944905

0.017180637

0.021963689

0.027234325

0.032927638

0.038977112

0.045317294

0.051885677

0.058623967

0.065478844

0.072402344

0.079351957

0.086290521

0.093185977

0.100011037

0.106742799

0.113362331

0.119854266

0.126206394

0.132409282

0.138455914

$\underline{0.144341366}$

0.056947951
(8)

$\begin{array}{cc}|(2)-(4)| & |(2)-(5)| \\ \underline{(b p s)} & \underline{(b p s)} \\ 0.000142401 & 0.000006624 \\ 0.001056683 & 0.000090133 \\ 0.003298451 & 0.000389281 \\ 0.007216019 & 0.001052843 \\ 0.012987996 & 0.002206282 \\ 0.020661140 & 0.003938545 \\ 0.030184757 & 0.006300063 \\ 0.041440047 & 0.009306489 \\ 0.054264031 & 0.012944905 \\ 0.068468357 & 0.017180637 \\ 0.083853570 & 0.021963689 \\ 0.100219580 & 0.027234325 \\ 0.117373021 & 0.032927638 \\ 0.135132168 & 0.038977112 \\ 0.153329950 & 0.045317294 \\ 0.171815551 & 0.051885677 \\ 0.190454964 & 0.058623967 \\ 0.209130815 & 0.065478844 \\ 0.227741673 & 0.072402344 \\ 0.246201054 & 0.079351957 \\ 0.264436220 & 0.086290521 \\ 0.282386903 & 0.093185977 \\ 0.300004003 & 0.100011037 \\ 0.317248314 & 0.106742799 \\ 0.334089319 & 0.113362331 \\ 0.350504061 & 0.119854266 \\ 0.366476107 & 0.126206394 \\ 0.381994613 & 0.132409282 \\ 0.397053476 & 0.138455914 \\ 0.411650592 & \underline{0.144341366} \\ 0.176027195 & 0.056947951\end{array}$


Table 9: GMM: Vasicek and Jump-Diffusion Models ( $J$ Distribution: Gaussian)

(Corresponding p values are listed under each parameter estimate.)

\begin{tabular}{|c|c|c|c|c|c|c|}
\hline & (1) & (2) & (3) & (4) & (5) & (6) \\
\hline Model: & Vasicek & Vasicek & Vasicek & Jumps: & Jumps: & Jumps: \\
\hline & & & & Gaussian & Gaussian & Gaussian \\
\hline Proxy: & Fed Funds & 1-month bill & 3-month bill & Fed Funds & 1-month bill & 3-month bill \\
\hline \multicolumn{7}{|l|}{ Parameter } \\
\hline \multirow[t]{2}{*}{$\mathrm{a}$} & 2.35281 & 4.14128 & 2.07811 & 3.05483 & 1.98156 & 2.46512 \\
\hline & 0.00000 & 0.00000 & 0.00000 & 0.00000 & 0.11304 & 0.00000 \\
\hline \multirow[t]{2}{*}{ b } & 0.04786 & 0.04229 & 0.04434 & 0.05037 & 0.01716 & 0.04325 \\
\hline & 0.00068 & 0.00242 & 0.00156 & 0.00000 & 0.49978 & 0.00000 \\
\hline \multirow[t]{2}{*}{$\sigma$} & 0.04938 & 0.05536 & 0.04088 & 0.05626 & 0.03614 & 0.04453 \\
\hline & 0.47219 & 0.48025 & 0.47850 & 0.47220 & 0.49990 & 0.47851 \\
\hline \multirow[t]{2}{*}{ h } & & & & 48.42087 & 18.09705 & 34.83129 \\
\hline & & & & 0.00000 & 0.00000 & 0.00000 \\
\hline \multirow[t]{2}{*}{$\alpha$} & & & & -0.00016 & 0.00275 & 0.00008 \\
\hline & & & & 0.44138 & 0.49967 & 0.47120 \\
\hline \multirow[t]{2}{*}{$\gamma$} & & & & 0.00000 & 0.00112 & 0.00000 \\
\hline & & & & 0.49943 & 0.50000 & 0.49938 \\
\hline Omnibus Test & 7.48E-09 & $7.41 \mathrm{E}-11$ & $1.64 \mathrm{E}-10$ & 7.48E-09 & 3.32E-11 & $1.64 \mathrm{E}-10$ \\
\hline
\end{tabular}


Table 10: GMM: Jump-Diffusion Models ( $J$ Distributions: Bernoulli Mixture of Gaussian Distributions)

(Corresponding p values are listed under each parameter estimate.)

\begin{tabular}{|c|c|c|c|c|c|c|}
\hline \multirow{3}{*}{ Model: } & (1) & (2) & (3) & (4) & (5) & (6) \\
\hline & Jumps: & Jumps: & Jumps: & $\begin{array}{l}\text { Jumps: } \\
\text { Gaussian }\end{array}$ & $\begin{array}{l}\text { Jumps: } \\
\text { Gaussian }\end{array}$ & $\begin{array}{l}\text { Jumps: } \\
\text { Gaussian }\end{array}$ \\
\hline & $\begin{array}{c}\text { Gaussian } \\
\text { Mixture }\end{array}$ & $\begin{array}{l}\text { Gaussian } \\
\text { Mixture }\end{array}$ & $\begin{array}{c}\text { Gaussian } \\
\text { Mixture }\end{array}$ & $\begin{array}{c}\text { Mixture } \\
\text { ("restricted") }\end{array}$ & $\begin{array}{c}\text { Mixture } \\
\text { ("restricted") }\end{array}$ & $\begin{array}{l}\text { Mixture } \\
\text { ("restricted") }\end{array}$ \\
\hline Proxy: & Fed Funds & 1-month bill & 3-month bill & Fed Funds & 1-month bill & 3-month bill \\
\hline \multicolumn{7}{|l|}{ Parameter } \\
\hline \multirow[t]{2}{*}{ a } & 2.25080 & 3.42960 & 2.64880 & 2.71445 & 2.61863 & 2.84140 \\
\hline & 0.00000 & 0.00000 & 0.28542 & 0.38115 & 0.49716 & 0.00000 \\
\hline \multirow[t]{2}{*}{ b } & 0.06045 & 0.04304 & 0.06600 & 0.04786 & 0.04229 & 0.04434 \\
\hline & 0.48938 & 0.49828 & 0.49994 & 0.00068 & 0.00242 & 0.00156 \\
\hline \multirow[t]{2}{*}{$\sigma$} & 0.00000 & 0.03122 & 0.00000 & 0.00525 & 0.03632 & 0.04500 \\
\hline & 0.48475 & 0.49723 & 0.49997 & 0.49999 & 0.50000 & 0.41741 \\
\hline \multirow[t]{2}{*}{$\mathrm{h}$} & 68.02432 & 33.76647 & 76.48174 & 23.30055 & 25.57396 & 26.13419 \\
\hline & 0.00000 & 0.00000 & 0.00000 & 0.00000 & 0.00006 & 0.00000 \\
\hline \multirow[t]{2}{*}{$\mathrm{w}$} & 0.45357 & 0.32966 & 0.44591 & & & \\
\hline & 0.13320 & 0.20020 & 0.49828 & & & \\
\hline \multirow[t]{2}{*}{$\alpha_{1}$} & -0.00628 & -0.00814 & -0.00725 & 0.00841 & 0.00492 & 0.00316 \\
\hline & 0.49976 & 0.49991 & 0.49980 & 0.50000 & 0.50000 & 0.49682 \\
\hline \multirow[t]{2}{*}{$\gamma_{1}$} & 0.00000 & 0.00000 & 0.00000 & 0.00699 & 0.00000 & 0.00000 \\
\hline & 0.49997 & 0.49997 & 0.50000 & 0.50000 & 0.50000 & 0.49682 \\
\hline \multirow[t]{2}{*}{$\alpha_{2}$} & 0.00445 & 0.00389 & 0.00448 & & & \\
\hline & 0.49979 & 0.49990 & 0.49990 & & & \\
\hline \multirow[t]{2}{*}{$\gamma_{2}$} & 0.00319 & 0.00462 & -0.00001 & & & \\
\hline & 0.49996 & 0.49996 & 0.50000 & & & \\
\hline Omnibus Test & 6.35E-09 & $4.06 \mathrm{E}-11$ & $4.20 \mathrm{E}-05$ & 7.46E-09 & $7.50 \mathrm{E}-11$ & $1.65 \mathrm{E}-10$ \\
\hline
\end{tabular}


Table 11: GMM: Jump-Diffusion Models ( $J$ Distributions: Bernoulli Mixture of Uniform Distributions)

(Corresponding p values are listed under each parameter estimate.)

\begin{tabular}{|c|c|c|c|}
\hline & (1) & (2) & (3) \\
\hline \multirow[t]{3}{*}{ Model: } & Jumps: & Jumps: & Jumps: \\
\hline & Uniform & Uniform & Uniform \\
\hline & Mixture & Mixture & Mixture \\
\hline Proxy: & Fed Funds & 1-month bill & 3-month bill \\
\hline \multicolumn{4}{|l|}{$\underline{\text { Parameter }}$} \\
\hline \multirow[t]{2}{*}{ a } & 0.16053 & 1.30912 & 0.32272 \\
\hline & 0.45789 & 0.32845 & 0.00860 \\
\hline \multirow[t]{2}{*}{$\mathrm{b}$} & 0.05178 & 0.02492 & 0.05251 \\
\hline & 0.33120 & 0.49963 & 0.47449 \\
\hline \multirow[t]{2}{*}{$\sigma$} & 0.01896 & 0.02880 & 0.00738 \\
\hline & 0.49974 & 0.49996 & 0.49964 \\
\hline \multirow[t]{2}{*}{ h } & 103.50648 & 41.59538 & 163.09353 \\
\hline & 0.00000 & 0.00000 & 0.00000 \\
\hline \multirow[t]{2}{*}{$\mathrm{w}$} & -1.02471 & 0.54412 & 0.48470 \\
\hline & 0.00000 & 0.34951 & 0.10191 \\
\hline \multirow[t]{2}{*}{$\omega_{1}$} & -0.00308 & -0.00106 & -0.00245 \\
\hline & 0.49989 & 0.49999 & 0.49998 \\
\hline \multirow[t]{2}{*}{$v_{1}$} & 0.00033 & -0.00069 & 0.00054 \\
\hline & 0.49999 & 0.50000 & 0.50000 \\
\hline \multirow[t]{2}{*}{$\omega_{2}$} & -0.00036 & 0.00428 & 0.00150 \\
\hline & 0.49996 & 0.49999 & 0.49999 \\
\hline \multirow[t]{2}{*}{$v_{2}$} & -0.00104 & 0.00021 & 0.00022 \\
\hline & 0.49997 & 0.50000 & 0.50000 \\
\hline Omnibus Test & $1.29 \mathrm{E}-10$ & 3.32E-11 & 5.16E-11 \\
\hline
\end{tabular}


Table 12: MLE: Vasicek Model

(Corresponding p values are listed under each parameter estimate.)

\begin{tabular}{|c|c|c|c|c|c|c|}
\hline & (1) & (2) & (3) & (4) & (5) & (6) \\
\hline Proxy: & Fed Funds & 1-month bill & 3-month bill & Fed Funds & 1-month bill & 3-month bill \\
\hline Frequency: & Daily & Daily & Daily & Weekly & Weekly & Weekly \\
\hline \multicolumn{7}{|l|}{ Parameter } \\
\hline \multirow[t]{2}{*}{ a } & 1.56994 & 0.49782 & 0.08045 & 1.03269 & 0.45170 & 0.07396 \\
\hline & 0.00103 & 0.04946 & 0.20959 & 0.00236 & 0.05360 & 0.21317 \\
\hline \multirow[t]{2}{*}{ b } & 0.04640 & 0.04133 & 0.02791 & 0.04622 & 0.04066 & 0.02747 \\
\hline & 0.00000 & 0.00044 & 0.23425 & 0.00000 & 0.00058 & 0.24934 \\
\hline \multirow[t]{2}{*}{$\sigma$} & 0.03996 & 0.01932 & -0.00750 & 0.03278 & 0.01821 & -0.00722 \\
\hline & 0.00000 & 0.00000 & 0.00000 & 0.00000 & 0.00000 & 0.00000 \\
\hline Log-Likelihood & 20506 & 23562 & 27810 & 3685 & 4229 & 5087 \\
\hline Observations & 4476 & 4439 & 4447 & 927 & 927 & 927 \\
\hline
\end{tabular}


Table 13: MLE: Jump-Diffusion Model ( $J$ Distribution: Gaussian)

(Corresponding p values are listed under each parameter estimate.)

\begin{tabular}{|c|c|c|c|c|c|c|}
\hline & (1) & (2) & (3) & (4) & (5) & (6) \\
\hline Proxy: & Fed Funds & 1-month bill & 3-month bill & Fed Funds & 1-month bill & 3-month bill \\
\hline Frequency: & Daily & Daily & Daily & Weekly & Weekly & Weekly \\
\hline \multicolumn{7}{|l|}{$\underline{\text { Parameter }}$} \\
\hline \multirow[t]{2}{*}{ a } & 1.61898 & 1.61286 & 1.61286 & 2.78885 & 0.59672 & 1.61286 \\
\hline & 0.00000 & 0.00000 & 0.00000 & 0.00000 & 0.00002 & 0.00000 \\
\hline \multirow[t]{2}{*}{ b } & 0.04293 & 0.02152 & 0.02152 & 0.02245 & 0.02615 & 0.02152 \\
\hline & 0.00000 & 0.00000 & 0.00000 & 0.00000 & 0.00001 & 0.00000 \\
\hline \multirow[t]{2}{*}{$\sigma$} & 0.01448 & -0.00778 & -0.00778 & 0.01150 & 0.00687 & -0.00778 \\
\hline & 0.00000 & 0.00000 & 0.00000 & 0.00000 & 0.00000 & 0.00000 \\
\hline \multirow[t]{2}{*}{ q } & 0.16366 & 0.14543 & 0.14543 & 0.35115 & 0.31967 & 0.14543 \\
\hline & 0.00000 & 0.00000 & 0.00000 & 0.00000 & 0.00000 & 0.00021 \\
\hline \multirow[t]{2}{*}{$\alpha$} & -0.00024 & -0.00025 & -0.00025 & -0.00007 & 0.00053 & -0.00025 \\
\hline & 0.15669 & 0.25035 & 0.42941 & 0.41774 & 0.02369 & 0.44749 \\
\hline \multirow[t]{2}{*}{$\gamma$} & 0.00585 & 0.00500 & 0.00500 & 0.00616 & 0.00411 & 0.00500 \\
\hline & 0.00000 & 0.00000 & 0.00000 & 0.00000 & 0.00000 & 0.02732 \\
\hline Log-Likelihood & 22460 & 24967 & 27400 & 3766 & 4448 & 4787 \\
\hline Observations & 4476 & 4439 & 4447 & 927 & 927 & 927 \\
\hline
\end{tabular}


Table 14: MLE: Jump-Diffusion Model ( $J$ Distributions: Bernoulli Mixture of Gaussian Distributions)

(Corresponding p values are listed under each parameter estimate.)

\begin{tabular}{|c|c|c|c|c|c|c|}
\hline & (1) & (2) & (3) & (4) & (5) & (6) \\
\hline Proxy: & Fed Funds & 1-month bill & 3-month bill & Fed Funds & 1-month bill & 3-month bill \\
\hline Frequency: & Daily & Daily & Daily & Weekly & Weekly & Weekly \\
\hline \multicolumn{7}{|l|}{ Parameter } \\
\hline \multirow[t]{2}{*}{ a } & 0.71688 & 2.12454 & 2.61228 & 1.24005 & 1.44447 & 1.29228 \\
\hline & 0.00665 & 0.00000 & 0.00000 & 0.00002 & 0.00000 & 0.00000 \\
\hline \multirow[t]{2}{*}{ b } & 0.05572 & 0.04523 & 0.03313 & 0.03427 & 0.03919 & 0.04198 \\
\hline & 0.00028 & 0.00000 & 0.00000 & 0.00015 & 0.03016 & 0.02341 \\
\hline \multirow[t]{2}{*}{$\sigma$} & 0.02267 & 0.01322 & 0.00896 & 0.02191 & 0.01561 & 0.00581 \\
\hline & 0.00000 & 0.00000 & 0.00000 & 0.00000 & 0.00000 & 0.09275 \\
\hline \multirow[t]{2}{*}{ q } & 0.13740 & 0.13162 & 0.17538 & 0.11132 & 0.09746 & 0.53182 \\
\hline & 0.00000 & 0.00000 & 0.00000 & 0.00009 & 0.42527 & 0.39862 \\
\hline \multirow[t]{2}{*}{$\mathrm{w}$} & 0.45304 & 0.50015 & 0.46923 & 0.11618 & 0.47624 & 0.39409 \\
\hline & 0.00000 & 0.00000 & 0.03728 & 0.00822 & 0.42105 & 0.41600 \\
\hline \multirow[t]{2}{*}{$\alpha_{1}$} & -0.00661 & -0.00674 & -0.00787 & -0.01759 & -0.00618 & -0.00243 \\
\hline & 0.00000 & 0.00000 & 0.00000 & 0.00000 & 0.00003 & 0.01589 \\
\hline \multirow[t]{2}{*}{$\gamma_{1}$} & 0.00071 & 0.00030 & 0.00031 & 0.00076 & 0.00045 & -0.00005 \\
\hline & 0.00000 & 0.00000 & 0.04676 & 0.25163 & 0.40938 & 0.49737 \\
\hline \multirow[t]{2}{*}{$\alpha_{2}$} & 0.00256 & 0.00263 & 0.00368 & 0.00778 & 0.00195 & 0.00037 \\
\hline & 0.00000 & 0.00000 & 0.00000 & 0.00000 & 0.40011 & 0.46941 \\
\hline \multirow[t]{2}{*}{$\gamma_{2}$} & 0.00061 & 0.00096 & 0.00084 & 0.00202 & 0.00073 & 0.00074 \\
\hline & 0.00000 & 0.00000 & 0.00486 & 0.00000 & 0.40713 & 0.32206 \\
\hline Log-Likelihood & 20639 & 24250 & 26676 & 3791 & 4255 & 4912 \\
\hline Observations & 4476 & 4439 & 4447 & 927 & 927 & 927 \\
\hline
\end{tabular}


Table 15: MLE: Jump-Diffusion Model ( $J$ Distributions: "Restricted” Bernoulli Mixture of Gaussian Distributions)

(Corresponding p values are listed under each parameter estimate.)

\begin{tabular}{|c|c|c|c|c|c|c|}
\hline & (1) & (2) & (3) & (4) & (5) & (6) \\
\hline Proxy: & Fed Funds & 1-month bill & 3-month bill & Fed Funds & 1-month bill & 3-month bill \\
\hline Frequency: & Daily & Daily & Daily & Weekly & Weekly & Weekly \\
\hline \multicolumn{7}{|l|}{$\underline{\text { Parameter }}$} \\
\hline \multirow[t]{2}{*}{ a } & 1.14986 & 0.85354 & 1.61286 & -0.03390 & 0.05091 & 0.00972 \\
\hline & 0.00001 & 0.00035 & 0.00000 & 0.17805 & 0.00013 & 0.08271 \\
\hline \multirow[t]{2}{*}{ b } & 0.02886 & 0.01605 & 0.02152 & 0.04368 & 0.02851 & 0.04832 \\
\hline & 0.00013 & 0.07460 & 0.00000 & 0.08148 & 0.00001 & 0.00017 \\
\hline \multirow[t]{2}{*}{$\sigma$} & 0.02267 & -0.01356 & -0.00778 & 0.01000 & -0.00218 & 0.00123 \\
\hline & 0.00000 & 0.00000 & 0.00000 & 0.41459 & 0.00000 & 0.00000 \\
\hline \multirow[t]{2}{*}{$\mathrm{q}$} & 0.00610 & 0.14983 & 0.14543 & 0.20648 & 0.30919 & 0.35224 \\
\hline & 0.00000 & 0.00000 & 0.00000 & 0.49842 & 0.00000 & 0.00000 \\
\hline \multirow[t]{2}{*}{$\alpha_{1}$} & 0.00675 & 0.00376 & 0.00373 & 0.00278 & 0.00000 & 0.00000 \\
\hline & 0.00000 & 0.00000 & 0.00000 & 0.49559 & 0.00000 & 0.00000 \\
\hline \multirow[t]{2}{*}{$\gamma_{1}$} & 0.00061 & 0.00069 & 0.00050 & 0.00025 & 0.00419 & 0.00141 \\
\hline & 0.00000 & 0.00000 & 0.00000 & 0.49984 & 0.00000 & 0.00000 \\
\hline Log-Likelihood & 21147 & 24347 & 27165 & 3678 & 4446 & 5161 \\
\hline Observations & 4476 & 4439 & 4447 & 927 & 927 & 927 \\
\hline
\end{tabular}


Table 16: MLE with a Latent Factor: Vasicek and Jump-Diffusion Models

(Corresponding p values are listed under each parameter estimate.)

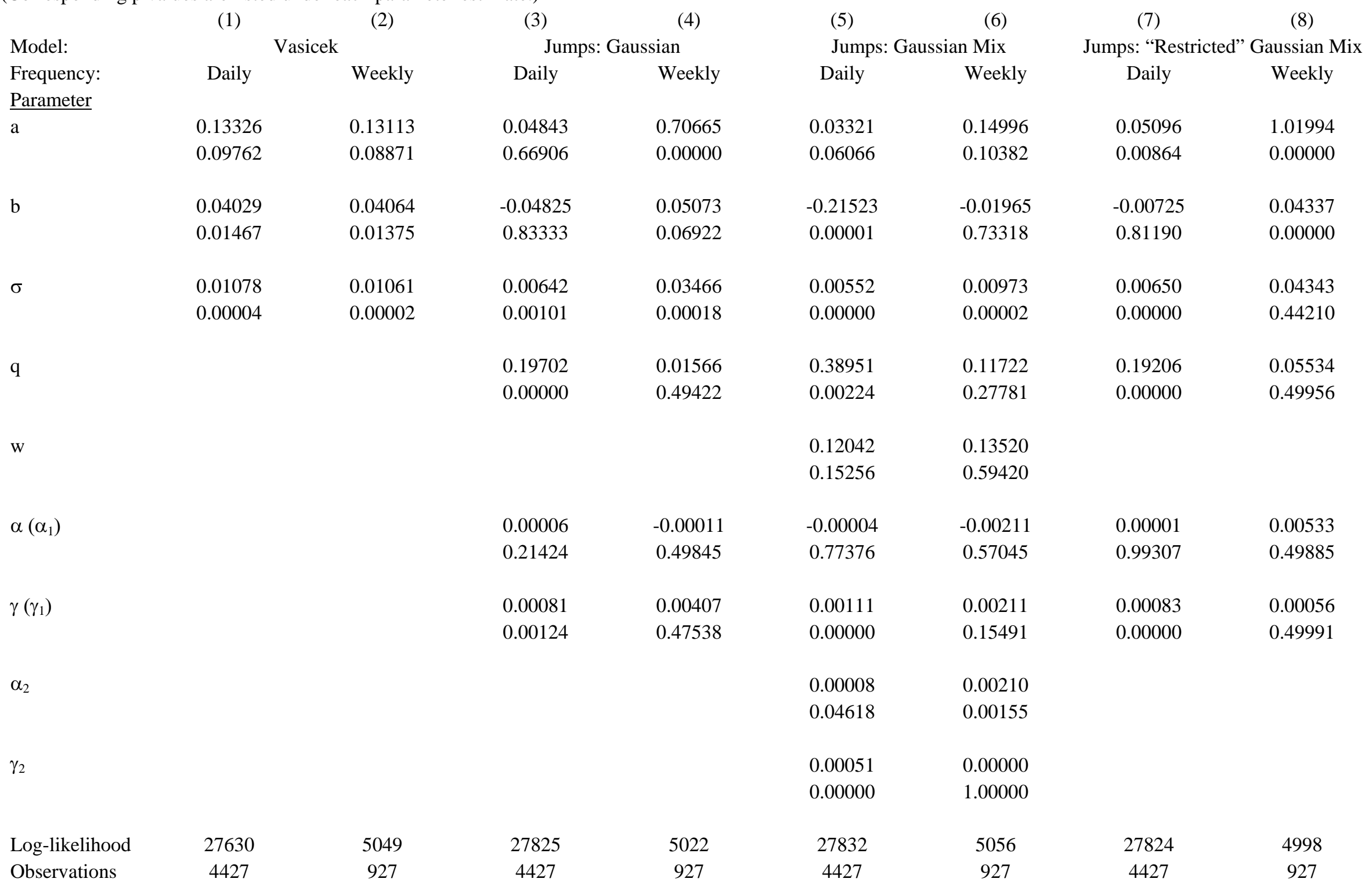


Table 17: MLE with a Latent Factor: Vasicek and Jump-Diffusion Models, Risk-Neutral Measure

(Corresponding p values are listed under each parameter estimate.)

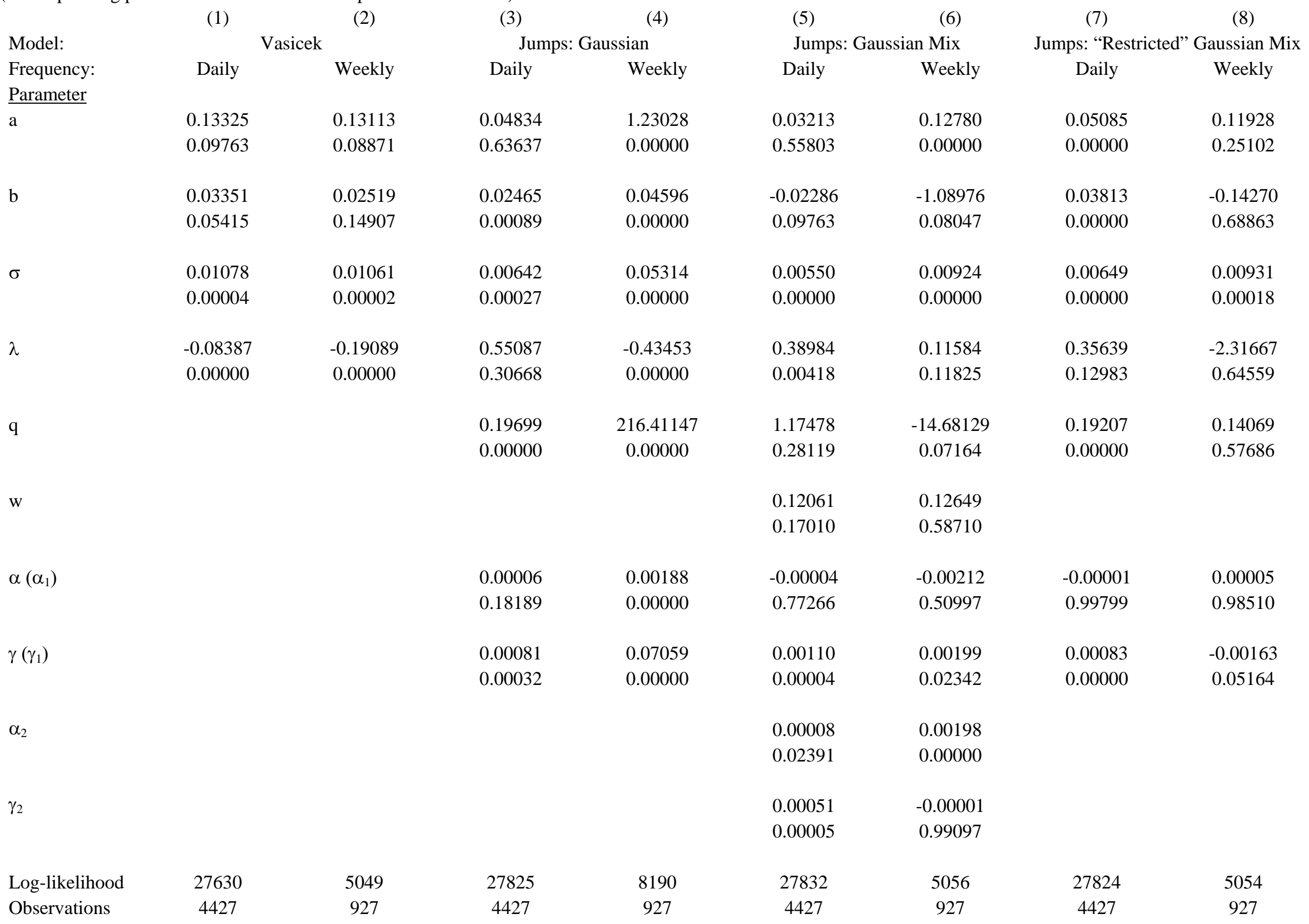


Table 18: MLE for Two-factor Model

(Corresponding p values are listed under each parameter estimate.)

\begin{tabular}{|c|c|c|c|c|}
\hline & (1) & (2) & (3) & (4) \\
\hline Stages: & One & One & Three & Three \\
\hline Frequency: & Daily & Weekly & Daily & Weekly \\
\hline \multicolumn{5}{|l|}{ Parameter } \\
\hline \multirow[t]{2}{*}{ a } & 1.49294 & 1.52500 & 0.08724 & 0.08882 \\
\hline & 0.00000 & 0.00000 & 0.25190 & 0.31094 \\
\hline \multirow[t]{2}{*}{$\sigma$} & 0.06423 & 0.05000 & 0.00741 & 0.00753 \\
\hline & 0.00000 & 0.00000 & 0.00000 & 0.00000 \\
\hline \multirow[t]{2}{*}{$\lambda_{\mathrm{r}}$} & -2.05534 & -0.05598 & $-3.62 \mathrm{E}-19$ & $-2.03 \mathrm{E}-0$ \\
\hline & 0.00000 & 0.98644 & 1.00000 & 1.00000 \\
\hline \multirow[t]{2}{*}{$q$} & 0.00001 & 0.25002 & 0.01690 & 0.01022 \\
\hline & 0.00000 & 0.80645 & 0.00002 & 0.27289 \\
\hline \multirow[t]{2}{*}{$\alpha_{1}$} & 0.13267 & 0.00500 & 0.00157 & 0.00259 \\
\hline & 0.00000 & 0.41533 & 0.00000 & 0.32128 \\
\hline \multirow[t]{2}{*}{$\gamma_{1}$} & 0.56456 & 0.00050 & 0.00076 & 0.00222 \\
\hline & 0.00000 & 0.98144 & 0.00001 & 0.06199 \\
\hline \multirow[t]{2}{*}{$\kappa$} & 1.13004 & 1.26250 & 1.64655 & 1.26287 \\
\hline & 0.00000 & 0.00000 & 0.00000 & 0.00000 \\
\hline \multirow[t]{2}{*}{$\beta$} & 0.07594 & 0.05153 & 0.02126 & 0.04495 \\
\hline & 0.00000 & 0.63033 & 0.00000 & 0.00000 \\
\hline \multirow[t]{2}{*}{$\phi$} & 0.00722 & 0.00500 & 0.00666 & 0.00500 \\
\hline & 0.00000 & 0.00000 & 0.00000 & 0.00000 \\
\hline \multirow[t]{2}{*}{$\lambda_{\mathrm{b}}$} & -12.25844 & -0.25500 & 0.00000 & -0.24235 \\
\hline & 0.00000 & 0.93069 & 1.00000 & 0.00000 \\
\hline Log-likelihood & 2291825 & 102121 & & \\
\hline Observations & 4427 & 927 & 4427 & 927 \\
\hline
\end{tabular}

